\title{
Species richness and distribution of bryophytes within different phytophysiognomies in the Chapada Diamantina region of Brazil ${ }^{1}$
}

\author{
Emilia de Brito Valente ${ }^{2,5}$, Kátia Cavalcanti Pôrto ${ }^{3}$ and Cid José Passos Bastos ${ }^{4}$
}

Submitted: 20 August, 2012. Accepted: 17 December, 2012

\begin{abstract}
The Chapada Diamantina ecoregion is within the caatinga (shrublands) biome of Brazil. Environmental factors determine the phytophysiognomies that distinguish the ecoregion from the surrounding areas. This study aimed to investigate the distribution of bryophyte flora in this ecoregion, by phytophysiognomy and elevational zone. Analyzing specimens we collected from five municipalities in the region, together with specimens (previously collected from the region) in herbaria, we identified 400 taxa. The phytophysiognomies that presented the highest species richness and the greatest numbers of exclusive taxa were forests and campos rupestres (dry, rocky grasslands), which respectively accounted for $51 \%$ and $40 \%$ of the taxa, compared with only $5 \%$ and $4 \%$, respectively, for the caatinga and cerrado (savanna). Species richness and the numbers of exclusive taxa were highest in the lower and upper montane zones. There was a predominance of neotropical taxa and a significant number of disjunct species found in Brazil and in the Andes region. We conclude that the Chapada Diamantina region is an important center of bryophyte diversity, harboring not only a great number of species overall but also a considerable number of species exclusive to the region, primarily in forests and campos rupestres at elevations above $800 \mathrm{~m}$.
\end{abstract}

Key words: Mosses, liverworts, forests, campos rupestres, elevational zonation

\section{Introduction}

The ecoregion of Chapada Diamantina, in the state of Bahia, Brazil, is one of the centers of plant diversity of the Americas (Giulietti et al. 1997). It is located within the caatinga (shrublands) biome (IBGE 2004), constituting one of its eight ecoregions (Velloso et al. 2002), and is entirely surrounded by the ecoregion of the Sertaneja Meridional Depression, the borders between the two being defined primarily by changes in physical aspects such as elevation, geological formation, climate, rainfall, topography and soil type (Rocha et al. 2005), which result in a mosaic of vegetation in the former (Giulietti \& Pirani 1988). According to the Brazilian Ministry of the Environment (MMA 2005), the vegetation in the Chapada Diamantina ecoregion comprises campos rupestres (dry, rocky grasslands), semi-deciduous montane forests, montane rain forests, cerrado (savanna) and caatinga.

Bryophytes, which represent the object of this study, are strongly influenced by external factors, particularly water and light, and differences in their physiognomy, composition, richness and abundance are evident among different vegetation formations and habitats (Mägdefrau, 1982). Numerous studies have indicated that bryophyte composition varies along an elevational gradient, showing greater diversity with increasing elevation (Van Reenen \& Gradstein, 1983, 1984; Frahm, 1990; Frahm \& Gradstein, 1991; Gradstein 1995; Kessler, 2000; Andrew et al., 2003; Ah-peng, 2007), this feature is also a consequence of high bryophyte sensitivity to climatic conditions.

In Brazil, the elevational zonation of bryophytes has been examined in two separate studies, both conducted in the Atlantic Forest within the state of Rio de Janeiro (Costa \& Lima, 2005; Santos \& Costa, 2010a). In both studies, the authors found that there is a variation in bryophyte flora composition along an elevational gradient, and that species richness, the number of exclusive taxa and the number of endemic taxa are highest in montane forests (sensu Veloso et al. 1991), followed by upper montane formations, lower montane formations and lowlands (sensu Veloso et al. 1991).

\footnotetext{
${ }^{1}$ Based on the Doctoral thesis of the first Author

${ }^{2}$ Universidade Estadual do Sudoeste da Bahia, Departamento de Ciências Naturais, Vitória da Conquista, BA, Brazil

${ }^{3}$ Universidade Federal de Pernambuco, Departamento de Botânica, Centro de Ciências Biológicas, Programa de Pós Graduação em Biologia Vegetal, Recife, PE, Brazil

${ }^{4}$ Universidade Federal da Bahia, Instituto de Biologia, Departamento de Botânica, Campus de Ondina, Salvador, BA, Brazil

${ }^{5}$ Author for correspondence: ebvalente@gmail.com
} 
As previously mentioned, the vegetation formations found in the Chapada Diamantina ecoregion include forests, campos rupestres, cerrado and caatinga. Among those, the highest bryophyte species richness and abundance is found in the forests. Therefore, in Brazil as a whole, systematic floristic studies of bryophytes have most often been conducted in forests, including those in the states of Rio de Janeiro (Costa 1999; Costa \& Lima 2005; Molinaro \& Costa 2001; Santos \& Costa 2010a, 2010b), Espírito Santo (Costa \& Silva 2003; Yano 2005), São Paulo (Visnadi 2005; Visnadi \& Vital 2000; Peralta \& Yano 2005), Pernambuco (Pôrto 1990, 1992; Germano \& Pôrto 1996; Campelo \& Pôrto 2007; Alvarenga \& Pôrto 2007), Alagoas (Silva \& Pôrto 2009) and Bahia (Valente \& Pôrto 2006; Bastos \& Valente 2008; Bastos \& Vilas Bôas-Bastos 2008; Vilas Bôas-Bastos \& Bastos 2008; Valente et al. 2009). Studies of bryophytes in the cerrado have been conducted in the states of São Paulo (Egunyomi \& Vital 1984; Visnadi 2004), Bahia (Vilas BôasBastos \& Bastos 1998) and Piauí (Castro et al. 2002), as well as in the Federal District of Brasília (Câmara \& Costa 2006; Câmara 2008a, 2008b). In the campos rupestres, bryophytes have been studied in the state of Minas Gerais-in the Serra do Cipó (Yano 1987), Serra da Piedade (Yano \& Carvalho 1995) and Grão Mogol (Yano \& Peralta 2009) — and in the state of Bahia, specifically within the Chapada Diamantina ecoregion (Harley 1995; Bastos et al. 1998a, 2000). Studies of bryophytes in the caatinga have been conducted in the states of Pernambuco (Pôrto et al. 1994) and Bahia (Bastos et al. 1998b).

The fact that there have been few studies of bryophytes in the Chapada Diamantina ecoregion is most likely attributable to the small number of researchers in this field of botany and the enormity of the region. The first contribution to knowledge of the bryophyte flora of this region was a species list compiled by Harley (1995), which included 28 species of liverworts and 37 species of mosses identified in the Pico das Almas region, near the municipality of Rio de Contas. Later, Bastos et al. (1998a) reported 27 species of mosses, collected in campos rupestres and in gallery forests near the town of Lençóis. In addition, Bastos et al. (2000) identified 65 species belonging to the divisions Bryophyta $(\mathrm{n}=41)$ and Marchantiophyta $(\mathrm{n}=24)$ in campos rupestres. Those authors stated that the majority of the bryophyte taxa surveyed in the Chapada Diamantina ecoregion have not been found elsewhere in the state of Bahia. A recently published Checklist of Bryophytes in the Chapada Diamantina region (Valente et al. 2011) lists 414 taxa, including various new records for the state of Bahia, for northeastern Brazil and for Brazil as a whole.

Considering the physical and climatic conditions, the phytophysiognomies within the Chapada Diamantina ecoregion and the bryophyte response to these conditions, this study aimed to investigate the distribution of the bryophyte flora in the phytophysiognomies and elevational zones in this ecoregion within the caatinga biome of Brazil.

\section{Material and methods}

\section{Study area}

The Chapada Diamantina ecoregion is $400 \mathrm{~km}$ in length and covers an area of 50,000 $\mathrm{km}^{2}$, representing approximately $9 \%$ of the State of Bahia. Elevations in the region range from $400 \mathrm{~m}$ to $2033 \mathrm{~m}$, making it the highest point in northeastern Brazil (MMA 2005). According to Nolasco et al. (2008), the average monthly temperature can be as low as $0^{\circ} \mathrm{C}$ in the winter (June through August) or as high as $30^{\circ} \mathrm{C}$ in the summer (in December and January). The rainy season is from November to April, with maximum rainfall in December $(139 \mathrm{~mm})$, and the dry season is from May to October, with minimum rainfall in August $(20 \mathrm{~mm})$. The average monthly rainfall exceeds $100 \mathrm{~mm}$ during the rainy season, compared with approximately $35 \mathrm{~mm}$ during the dry season. The average annual rainfall ranges from $600 \mathrm{~mm}$ to $1100 \mathrm{~mm}$ (Agritempo 2010). Geologically, the Chapada Diamantina ecoregion consists of quartzite and sandstone outcrops, and there are a number of rivers in the region (Rocha et al. 2005).

The type of phytophysiognomy that is the most characteristic of the Chapada Diamantina ecoregion is campos rupestres, which typically occur at elevations above $900 \mathrm{~m}$ and are characterized mainly by rocky outcrops associated with herb-shrub areas, typically on quartzite soils, the predominant species belonging to the families Velloziaceae, Melastomataceae, Eriocaulaceae, Xyridaceae and Orchidaceae (Conceição et al. 2005). Forest types, including semideciduous forest, premontane rain forest and montane rain forest, typically to the east of the main mountain range, also occur on plateaus (plateau forests), along the banks of rivers (riparian forests) and between large rocky cliffs (cove forests) (Funch et al. 2005; Funch 2008; Queiroz et al. 2008). Throughout the region, cerrado occurs at elevations of $900-1200 \mathrm{~m}$ and is interspersed with campos rupestres, where rocky outcrops and shallow soils appear more frequently. At lower elevations, the cerrado is replaced by various forms of dry forest or caatinga (Harley et al. 2005). Caatinga, or phytophysiognomies associated with it, cover the greatest area in the region, albeit quite diverse in its physiognomy, floristic composition and community structure, and is found mainly to the west of the main mountain range (Queiroz et al. 2005).

\section{Sampling}

Specimens were collected during 11 field campaigns conducted between 2007 and 2009 in the Chapada Diamantina ecoregion, in the municipalities of Morro do Chapéu and Miguel Calmon (located in the north of the region) Lençóis and Palmeiras (in the center); and Piatã and Abaíra (in the south). The municipalities were selected considering the preexisting knowledge about the diversity of other plant groups (MMA 2005) and the presence of the target phytophysiognomies and elevational zones. In each municipality, 
three to six sites were surveyed. The areas were walked for approximately seven hours, and the sampling effort was given by stabilization of the species accumulation curve. The samples collected were deposited in the Herbarium of the Estate University of Feira de Santana (code, HUEFS), with duplicates in the Herbarium of the Federal University of Pernambuco (code, UFP). We employed traditional methods of collection and preservation, as described by Yano (1989b).

The sampling sites were located across five ecologically protected areas (Chapada Diamantina National Park; the Marimbus/Iraquara Environmentally Protected Area; the Serra do Barbado Environmentally Protected Area, in the municipality of Abaíra; Sete Passagens State Park, in the municipality of Miguel Calmon; and the Cachoeira do Ferro Doido Monument), as well as in areas external to conservation units. National herbaria with representative collections of bryophyte of this region were consulted: the Alexandre Leal Costa Herbarium (code, ALCB), affiliated with the Federal University of Bahia Institute of Biology; the Herbarium of the Estate University of Feira de Santana (HUEFS), in the state of Bahia; the Herbarium of the Center for Cacao Research (CEPEC), in the state of Bahia; the Herbarium of the Botanical Institute of São Paulo (SP); and the University of São Paulo Herbarium of Phanerogamae (acronym, SPF). In addition, the species list was supplemented with data from the literature (Yano 1981, 1984, 1989a, 1995, 1996a, 2006, 2008; Yano \& Bastos 1994; Harley 1995; Bastos et al. 1998a, 2000; Yano \& Peralta 2006; Peralta \& Vital 2006; Ballejos \& Bastos 2009a, 2009b). Regardless of the source (herbaria or literature), we analyzed only those data related to samples for which there was information about the vegetation formation of origin or elevation. Overall, approximately 2300 samples were analyzed, covering nine cities within the Chapada Diamantina ecoregion: in the north (Morro do Chapéu, Miguel Calmon and Jacobina); in the south (Abaíra, Rio de Contas and Piatã); and in the center (Lençóis, Palmeiras and Mucugê).

\section{Study material and data analysis}

The taxa identification was based mainly on Crum (1984), Yano et al. (1985), Frahm (1991), Reese (1993), Zander (1993), Sharp et al. (1994), Buck (1998), Gradstein et al. (2001), Gradstein \& Costa (2003) and Pursell (2007). We adopted the classification systems presented by Goffinet et al. (2009) for mosses and by Crandall-Stotler et al. (2009) for liverworts. The taxa authority name abbreviations were based on Brummitt \& Powell (1992). The Brazilian state abbreviations are in accordance with the Brazilian Institute of Geography and Statistics guidelines. The geographic distribution of species was based on Yano (1981; 1984; 1989; 1995; 1996; 2006; 2008), Bastos \& Yano (2009), Ballejos \& Bastos (2009a; 2009b), and Forzza et al. (2012).

We performed a comparative analysis between the different bryophyte flora, based on the presence or absence of species, using the Bray-Curtis method and the software PRIMER 5.1 (Clarke \& Warwick 1994). To analyze the geographic distribution of the taxa in Brazil, we defined the following categories of species distribution: restricted, occurring in four of fewer states; moderate, occurring in five to nine states; and broad, occurring in ten or more states (Valente \& Pôrto 2006). For the analysis of the elevational distribution of the bryophyte flora from the Chapada Diamantina ecoregion, we used the elevational zones established by Oliveira-Filho et al. (2006) for latitudes $<16^{\circ}$ S, in which lowland formations are defined as occurring at elevations $\leq 400 \mathrm{~m}$; premontane formations are defined as occurring at elevations of 400-800 m; lower montane formations are defined as occurring at elevations of 800-1200 m; and upper montane formations are defined as occurring at elevations $>1200 \mathrm{~m}$. However, we evaluated only the last three of those zones.

\section{Results and discussion}

We identified a total of 400 taxa (Tab. 1). Moss species predominated over liverworts in all phytophysiognomies, except in the forest. Species richness and the number of exclusive taxa were greatest in the forests ( 272 species, 157 exclusive), followed by campos rupestres (212 species, 93 exclusive), caatinga (29 species, 18 exclusive) and Cerrado (20 species, three exclusive). This result was predictable, because the bryophyte composition and richness reflect the environmental conditions in each phytophysiognomy. We should point out that the caatinga and the cerrado, when compared with the forest and the campos rupestres, present conditions that are much more restrictive for the development and establishment of most bryophyte species, mainly due to low humidity and high light intensity. Although the campos rupestres could also be considered restrictive, humidity from the frequent occurrence of fog and from localized accumulations of water, as well as the quite rugged topography, in which there are numerous rock clefts, provides a myriad of microhabitats that are favorable to the growth of the bryophyte flora adapted to those conditions.

As a result of the analysis of bryophyte flora similarity among the phytophysiognomies, we identified clusters in which the forests and campos rupestres shared $46 \%$ of the species, compared with only $15 \%$ for the cerrado and caatinga. This might be explained by the previously mentioned factors (higher humidity presence and shaded microhabitats) in forests and campos rupestres.

In the forests, Lejeuneaceae, Plagiochilaceae, Radulaceae, Leucobryaceae, Sematophyllaceae and Orthotrichaceae were the most representative families in terms of the number of species. These families are typical of tropical rain forests (Pócs 1982; Richards 1984; Gradstein \& Pócs 1989; Gradstein 1995; Gradstein et al. 2001). The majority of their constituents are adapted to the low-light conditions, like most bryophytes, with leaves consisting of a single layer of 
Species richness and distribution of bryophytes within different phytophysiognomies in the Chapada Diamantina region of Brazil

Table 1. Distribution of Bryophyte species in the Chapada Diamantina ecoregion, in the state of Bahia, Brazil, by elevational zone and phytophysiognomy.

\begin{tabular}{|c|c|}
\hline Species & Elevational zone/phytophysiognomy \\
\hline Acrolejeunea emergens (Mitt.) Steph. & $\mathrm{LM} / \mathrm{cr}$, ce \\
\hline Acrolejeunea torulosa (Lehm. \& Lindenb.) Schiffn. & $\mathrm{LM} / \mathrm{cr}$, ce \\
\hline Acroporium caespitosum (Hedw.) W.R. Buck & $\mathrm{LM} / \mathrm{cr}, \mathrm{f}$ \\
\hline Acroporium estrellae (Müll. Hal.) W.R. Buck \& Schäf.-Verw. & $\mathrm{UM}, \mathrm{LM} / \mathrm{cr}, \mathrm{f}$ \\
\hline Acroporium pungens (Hedw.) Broth. & $-/ c r, f$ \\
\hline Adelanthus decipiens (Hook.) Mitt. & $-/ \mathrm{cr}$ \\
\hline Adelothecium bogotense (Hampe) Mitt. & $\mathrm{UM} / \mathrm{cr}, \mathrm{f}$ \\
\hline Anastrophyllum piligerum (Nees) Steph. & $\mathrm{UM} / \mathrm{cr}$ \\
\hline Anoplolejeunea conferta (Meissn.) A. Evans & $\mathrm{UM}, \mathrm{LM} / \mathrm{cr}, \mathrm{f}$ \\
\hline Aphanolejeunea asperrima (Stephani) Steph. & $\mathrm{UM} /$ \\
\hline Aphanolejeunea cornutissima R.M. Schust. & $\mathrm{UM} / \mathrm{f}$ \\
\hline Aptychopsis pyrrophylla (Müll. Hal.) Wijk \& Marg. & $\mathrm{UM}, \mathrm{LM} / \mathrm{cr}, \mathrm{f}$ \\
\hline Archidium clavatum I.G. Stone & $\mathrm{LM} / \mathrm{ca}$ \\
\hline Archidium donnellii Aust. & $\mathrm{LM} / \mathrm{ca}$ \\
\hline Archidium ohioense Schimp. ex Müll. Hal. & $\mathrm{LM} / \mathrm{ca}$ \\
\hline Atractylocarpus brasiliensis (Müll. Hal.) R.S. Williams & $\mathrm{LM} / \mathrm{f}$ \\
\hline Bazzania aurescens Spruce & $\mathrm{LM} / \mathrm{f}$ \\
\hline Bazzania falcata (Lindenb.) Trevis. & $\mathrm{UM} / \mathrm{cr}$ \\
\hline Bazzania heterostipa (Steph.) Fulford & $\mathrm{UM}, \mathrm{LM} / \mathrm{cr}, \mathrm{f}$ \\
\hline Bazzania hookeri (Lindenb.) Trevis. & $\mathrm{UM} / \mathrm{f}$ \\
\hline Bazzania nitida (Web.) Grolle & $\mathrm{UM} / \mathrm{cr}$ \\
\hline Bazzania stolonifera (Sw.) Trevis. & $\mathrm{UM} / \mathrm{cr}$ \\
\hline Brachiolejeunea leiboldiana (Gott. \& Lindenb.) Schiffn. & $\mathrm{UM}, \mathrm{LM} / \mathrm{cr}, \mathrm{f}$ \\
\hline Brachymenium systylium (Müll. Hal.) A. Jaeger & $\mathrm{LM} / \mathrm{ca}$ \\
\hline Breutelia tomentosa (Sw. ex Brid.) A. Jaeger & $\mathrm{LM} / \mathrm{cr}$ \\
\hline Bryopteris diffusa (Sw.) Nees & $\mathrm{LM} / \mathrm{cr}, \mathrm{f}$ \\
\hline Bryum argenteum Hedw. & $\mathrm{UM}, \mathrm{LM}, \mathrm{PM} / \mathrm{cr}$ \\
\hline Bryum limbatum Müll. Hal. & $\mathrm{UM}, \mathrm{LM} / \mathrm{cr}, \mathrm{f}$ \\
\hline Bryum paradoxum Schwägr. & $\mathrm{UM} / \mathrm{cr}$ \\
\hline Callicostella merkelii (Hornsch.) A. Jaeger & $\mathrm{LM} / \mathrm{cr}$ \\
\hline Callicostella pallida (Hornsch.) Ångstr. & $\mathrm{LM} / \mathrm{cr}$ \\
\hline Callicostella rufescens (Mitt.) A. Jaeger & $\mathrm{LM} / \mathrm{f}$ \\
\hline Calymperes palisotii Schwägr. & $\mathrm{LM} / \mathrm{cr}$ \\
\hline Calypogeia andicola Bischl. & LM \\
\hline Calypogeia laxa Lindenb. \& Gottsche & $\mathrm{UM} / \mathrm{cr}, \mathrm{f}$ \\
\hline Calypogeia peruviana Nees & $\mathrm{UM} / \mathrm{f}$ \\
\hline Campylopus arctocarpus (Hornsch.) Mitt. & $\mathrm{UM}, \mathrm{LM} / \mathrm{cr}$ \\
\hline Campylopus arctocarpus var. caldense (Angström) J.-P. Frahm & $\mathrm{UM} / \mathrm{cr}$ \\
\hline Campylopus cf. subcuspidatus (Hampe) A. Jaeger & $\mathrm{UM} / \mathrm{cr}$ \\
\hline Campylopus controversus (Hampe) A. Jaeger & $-/ \mathrm{cr}$ \\
\hline Campylopus cuspidatus (Hornsch.) Mitt. & $\mathrm{UM} / \mathrm{cr}$ \\
\hline Campylopus dichrotis Paris & $\mathrm{LM} / \mathrm{cr}$ \\
\hline Campylopus filifolius (Hornsch.) Mitt. & $\mathrm{UM}, \mathrm{LM} / \mathrm{cr}, \mathrm{f}$ \\
\hline Campylopus filifolius var. humilis (Mont.) J.-P. Frahm & $\mathrm{UM} / \mathrm{cr}, \mathrm{f}$ \\
\hline
\end{tabular}


Table 1. Continuation.

\begin{tabular}{|c|c|}
\hline Species & Elevational zone/phytophysiognomy \\
\hline Campylopus filifolius var. longifolius (E.B. Bartram) E.B. Bartram & $\mathrm{UM}, \mathrm{LM} / \mathrm{cr}, \mathrm{f}$ \\
\hline Campylopus fragilis (Brid.) Bruch \& Schimp. & $\mathrm{UM}, \mathrm{LM}, \mathrm{PM} / \mathrm{cr}$ \\
\hline Campylopus heterostachys (Hampe) A. Jaeger & $\mathrm{UM}, \mathrm{LM} / \mathrm{cr}$ \\
\hline Campylopus introflexus (Hedw). Brid. & $\mathrm{LM} / \mathrm{cr}$ \\
\hline Campylopus julaceus A. Jaeger & $\mathrm{UM}, \mathrm{LM} / \mathrm{cr}, \mathrm{f}$ \\
\hline Campylopus julicaulis Broth. & $\mathrm{LM} /-$ \\
\hline Campylopus lamellinervis (Müll. Hal.) Mitt. & $\mathrm{UM} / \mathrm{cr}, \mathrm{f}$ \\
\hline Campylopus lamellinervis var. exaltatus (Müll. Hal.) J.-P. Frahm & $-/ F$ \\
\hline Campylopus occultus Mitt. & $\mathrm{UM}, \mathrm{LM}, \mathrm{PM} / \mathrm{cr}$ \\
\hline Campylopus pilifer Brid. & $\mathrm{UM}, \mathrm{LM}, \mathrm{PM} / \mathrm{cr}$ \\
\hline Campylopus richardii Brid. & $\mathrm{LM} /-$ \\
\hline Campylopus savannarum (Müll. Hal.) Mitt. & $\mathrm{UM}, \mathrm{LM}, \mathrm{PM} / \mathrm{cr}, \mathrm{f}, \mathrm{ce}, \mathrm{ca}$ \\
\hline Campylopus surinamensis Müll. Hal. & $\mathrm{UM} / \mathrm{cr}$ \\
\hline Campylopus trachyblepharon (Müll. Hal.) Mitt. & $\mathrm{LM} / \mathrm{cr}, \mathrm{f}, \mathrm{ca}$ \\
\hline Campylopus uleanus (Müll. Hal.) Broth. & $-/ \mathrm{cr}$ \\
\hline Campylopus viridatus (Müll. Hal.) Broth. & $-/ \mathrm{cr}$ \\
\hline Campylopus widgrenii (Müll. Hal.) Mitt. & $\mathrm{UM}, \mathrm{LM} / \mathrm{cr}$ \\
\hline Cardotiella quinquefaria (Hornsch.) Vitt & $\mathrm{UM} /-$ \\
\hline Cephaloziella cf. granatensis (J.B. Jack) Fulford & $\mathrm{LM} / \mathrm{f}$ \\
\hline Cephaloziopsis intertexta (Gottsche) R.M. Schust. & LM, PM/- \\
\hline Ceratolejeunea guianensis (Nees \& Mont.) Steph. & $\mathrm{LM} / \mathrm{cr}$ \\
\hline Ceratolejeunea laetefusca (Austin) R.M. Schust. & $\mathrm{UM} / \mathrm{f}$ \\
\hline Cheilolejeunea acutangula (Nees) Grolle & $\mathrm{UM} / \mathrm{f}$ \\
\hline Cheilolejeunea discoidea (Lenm \& Lindenb.) Kachroo \& R.M. Schust. & $\mathrm{LM} / \mathrm{ce}$ \\
\hline Cheilolejeunea holostipa (Spruce) Grolle \& R.L. Zhu & $\mathrm{UM} / \mathrm{f}$ \\
\hline Cheilolejeunea oncophylla (Ångstr.) Grolle \& M.E. Reiner & $\mathrm{UM}, \mathrm{LM} / \mathrm{cr}, \mathrm{f}$ \\
\hline Cheilolejeunea rigidula (Mont.) R.M. Schust. & $\mathrm{UM}, \mathrm{LM} / \mathrm{f}, \mathrm{ca}$ \\
\hline Cheilolejeunea trifaria (Reinw., Blume \& Nees) Mizut. & $\mathrm{LM} / \mathrm{cr}, \mathrm{f}$ \\
\hline Cheilolejeunea unciloba (Lindenb.) Malombe & $\mathrm{UM}, \mathrm{LM} / \mathrm{f}$ \\
\hline Cheilolejeunea xanthocarpa (Lehm. \& Lindenb.) Malombe & $\mathrm{UM}, \mathrm{LM} / \mathrm{cr}, \mathrm{f}$ \\
\hline Chiloscyphus bidentatus Steph. & $\mathrm{UM} / \mathrm{f}$ \\
\hline Chiloscyphus latifolius (Nees) J.J. Engel \& R.M. Schust. & $\mathrm{UM} / \mathrm{cr}$ \\
\hline Chiloscyphus martianus (Nees) J.J. Engel \& R.M. Schust. & $\mathrm{UM}, \mathrm{LM} / \mathrm{cr}, \mathrm{f}$ \\
\hline Chiloscyphus martianus subsp. bidentulus Nees & $\mathrm{UM} / \mathrm{cr}, \mathrm{f}$ \\
\hline Colobodontium vulpinum (Mont.) S.P. Churchill \& W.R. Buck & $\mathrm{UM}, \mathrm{LM} / \mathrm{cr}$ \\
\hline Cololejeunea cf. hildebrandii (Austin) Steph. & $\mathrm{UM} / \mathrm{f}$ \\
\hline Cololejeunea minutissima (Sm.) Schiffin. & $\mathrm{LM} / \mathrm{ce}$ \\
\hline Cololejeunea subcardiocarpa Tixier & $\mathrm{UM} / \mathrm{f}$ \\
\hline Colura tenuicornis (A. Evans) Steph. & $\mathrm{UM} / \mathrm{f}$ \\
\hline Cronisia weddellii (Mont.) Grolle & $\mathrm{LM} / \mathrm{ca}$ \\
\hline Ctenidium malacodes Mitt. & $-/ f$ \\
\hline Cyclolejeunea convexistipa (Lehm. ex. Lindenb.) A. Evans & $\mathrm{LM} / \mathrm{f}$ \\
\hline Cyclolejeunea luteola (Spruce) Grolle & $\mathrm{LM} / \mathrm{f}$ \\
\hline Cylindrocolea planifolia (Steph.) R.M. Schust. & $\mathrm{UM} / \mathrm{f}$ \\
\hline
\end{tabular}


Table 1. Continuation.

\begin{tabular}{|c|c|}
\hline Species & Elevational zone/phytophysiognomy \\
\hline Cylindrocolea rhizantha (Mont.) R.M. Schust. & $\mathrm{LM} / \mathrm{f}$ \\
\hline Daltonia gracilis Mitt. & $\mathrm{UM}, \mathrm{LM} / \mathrm{cr}, \mathrm{f}$ \\
\hline Daltonia longifolia Taylor & $\mathrm{UM} / \mathrm{f}$ \\
\hline Dicranella cf. harrisii (Müll. Hal.) Broth. & $\mathrm{UM} / \mathrm{f}$ \\
\hline Dicranodontium pulchroalare subsp. brasiliense (Herzog) J.-P. Frahm & $\mathrm{UM} / \mathrm{f}$ \\
\hline Diplasiolejeunea latipuense Tixier & $\mathrm{LM} / \mathrm{f}$ \\
\hline Diplasiolejeunea pellucida (C.F.W. Meissn. ex Spreng.) Schiffn. & $\mathrm{LM} / \mathrm{f}$ \\
\hline Diplasiolejeunea rudolphiana Steph. & $\mathrm{PM} / \mathrm{f}$ \\
\hline Diplasiolejeunea unidentata (Lehm. \& Lindenb.) Steph. & $\mathrm{UM} / \mathrm{f}$ \\
\hline Donnellia commutata (Müll. Hal.) W.R. Buck & $\mathrm{UM}, \mathrm{LM} / \mathrm{cr}, \mathrm{f}$ \\
\hline Drepanolejeunea anoplantha (Spruce) Steph. & $\mathrm{UM} / \mathrm{cr}, \mathrm{f}$ \\
\hline Drepanolejeunea araucariae Steph. & $\mathrm{UM}, \mathrm{LM} / \mathrm{f}$ \\
\hline Drepanolejeunea campanulata (Spruce) Steph. & $\mathrm{UM} / \mathrm{cr}$ \\
\hline Drepanolejeunea fragilis Bischl. & $\mathrm{UM}, \mathrm{LM}, \mathrm{PM} / \mathrm{f}$ \\
\hline Drepanolejeunea mosenii (Steph.) Bischl. & $\mathrm{LM} / \mathrm{f}$ \\
\hline Drepanolejeunea orthophylla Bischl. & $\mathrm{UM} / \mathrm{f}$ \\
\hline Eccremidium floridanum H.A. Crum & LM, PM/- \\
\hline Ectropothecium leptochaeton (Schwäegr.) W.R. Buck & $\mathrm{LM} / \mathrm{f}$ \\
\hline Entodon macropodus (Hedw.) Müll. Hal. & $\mathrm{LM}, \mathrm{PM} / \mathrm{cr}, \mathrm{f}$ \\
\hline Entodontopsis leucostega (Brid.) W.R. Buck \& Ireland & $\mathrm{LM} / \mathrm{cr}$, ce \\
\hline Erpodium biseriatum (Austin) Austin & $-/ f$ \\
\hline Erythrodontium squarrosum (Hampe) Paris & $-/ f$ \\
\hline Fabronia ciliaris (Brid.) Brid. & $\mathrm{PM} / \mathrm{cr}$, ce \\
\hline Fabronia ciliaris var. polycarpa (Hook.) W.R. Buck & $\mathrm{PM} / \mathrm{ce}$ \\
\hline Fabronia macroblepharis Schwägr. & $\mathrm{UM}, \mathrm{LM} / \mathrm{cr}$, ce \\
\hline Fissidens elegans Brid. & $-/ \mathrm{cr}$ \\
\hline Fissidens pellucidus Hornsch. & $\mathrm{UM}, \mathrm{LM} / \mathrm{f}$ \\
\hline Fissidens ramicola Broth. & $\mathrm{LM} / \mathrm{ca}$ \\
\hline Fissidens serratus Müll. Hal. & $-/ f$ \\
\hline Fissidens termitarum (Herzog) Pursell & $\mathrm{LM} / \mathrm{ca}$ \\
\hline Fissidens weirii var. hemicraspedophyllus (Cardot) Pursell & $\mathrm{UM} / \mathrm{f}$ \\
\hline Floribundaria flaccida (Mitt.) Broth. & $-/ \mathrm{cr}$ \\
\hline Fossombronia porphyrorhiza (Nees) Prosk. & $\mathrm{UM}, \mathrm{LM} / \mathrm{cr}, \mathrm{ca}$ \\
\hline Frullania arecae (Spreng.) Gottsche & $\mathrm{UM} / \mathrm{f}$ \\
\hline Frullania atrata (Sw.) Dumort. & $\mathrm{UM}, \mathrm{LM} / \mathrm{f}$ \\
\hline Frullania beyrichiana (Lehm. \& Lindenb.) Lehm. \& Lindenb. & $\mathrm{UM}, \mathrm{LM} / \mathrm{cr}, \mathrm{f}$ \\
\hline Frullania brasiliensis Raddi & $\mathrm{UM}, \mathrm{LM} / \mathrm{cr}, \mathrm{f}$ \\
\hline Frullania breuteliana Gottsche & $\mathrm{UM} / \mathrm{f}$ \\
\hline Frullania caulisequa (Nees) Nees & $\mathrm{UM} / \mathrm{f}$, ce \\
\hline Frullania ericoides (Nees ex Mart.) Mont. & $\mathrm{LM} / \mathrm{ce}, \mathrm{ca}$ \\
\hline Frullania gibbosa Nees & $\mathrm{LM} / \mathrm{cr}$, ca \\
\hline Frullania glomerata (Lehm. \& Lindenb.) Nees \& Mont. & $\mathrm{LM} / \mathrm{ca}$ \\
\hline Frullania griffithsiana Gottsche & $\mathrm{UM} / \mathrm{cr}, \mathrm{f}$ \\
\hline Frullania kunzei Lehm. \& Lindenb. & $\mathrm{UM}, \mathrm{LM} / \mathrm{cr}, \mathrm{f}, \mathrm{ce}$ \\
\hline
\end{tabular}


Table 1. Continuation.

\begin{tabular}{|c|c|}
\hline Species & Elevational zone/phytophysiognomy \\
\hline Frullania lindenbergii Lehm. & $\mathrm{UM} / \mathrm{f}$ \\
\hline Frullania mucronata (Lehm. \& Lindenb.) Lehm. \& Lindenb. & $\mathrm{UM}, \mathrm{LM} / \mathrm{f}$ \\
\hline Frullania riojaneirensis (Raddi) Spruce & $\mathrm{LM} / \mathrm{cr}$ \\
\hline Frullania setigera Steph. & $\mathrm{UM} / \mathrm{cr}, \mathrm{f}$ \\
\hline Frullanoides densifolia Raddi & $\mathrm{UM} / \mathrm{f}$ \\
\hline Funaria hygrometrica Hedw. & $\mathrm{UM} / \mathrm{cr}$ \\
\hline Funaria hygrometrica var. calvescens (Schwägr.) Mont. & $\mathrm{UM} / \mathrm{cr}$ \\
\hline Gemmabryum coronatum Schwägr. & $\mathrm{UM}, \mathrm{LM}, \mathrm{PM} / \mathrm{cr}$ \\
\hline Gemmabryum exile (Dozy \& Molk.) J.R. Spence \& H.P. Ramsay & $\mathrm{UM}, \mathrm{LM} / \mathrm{cr}, \mathrm{f}$ \\
\hline Gemmabryum radiculosum (Brid.) J.R. Spence \& H.P. Ramsay & $\mathrm{UM}, \mathrm{LM} / \mathrm{cr}$ \\
\hline Groutiella apiculata (Hook.) H. A. Crum \& Steere & $-/ \mathrm{f}$ \\
\hline Groutiella tomentosa (Hornsch.) Wijk \& Margad. & $\mathrm{LM} / \mathrm{cr}$ \\
\hline Groutiella tumidula (Mitt.) Vitt & $\mathrm{LM} /-$ \\
\hline Harpalejeunea schiffneri S. Arnell & $\mathrm{UM} / \mathrm{f}$ \\
\hline Harpalejeunea stricta Schiffn. & $\mathrm{UM} / \mathrm{f}$ \\
\hline Harpalejeunea subacuta A. Evans & $\mathrm{UM} / \mathrm{f}$ \\
\hline Helicophyllum torquatum (Hook.) Brid. & $-/ \mathrm{ca}$ \\
\hline Herbertus juniperoideus (Swartz) Grolle & $-/ \mathrm{cr}$ \\
\hline Herbertus juniperoideus subsp. bivittatus (Spruce) Feldberg \& J. Heinrichs & $\mathrm{UM}, \mathrm{LM} / \mathrm{f}$ \\
\hline Holomitrium arboreum Mitt. & $\mathrm{UM}, \mathrm{LM} / \mathrm{cr}$ \\
\hline Holomitrium crispulum Mart. & $\mathrm{UM}, \mathrm{LM} / \mathrm{cr}$ \\
\hline Holomitrium olfersianum Hornsch. & $\mathrm{UM}, \mathrm{LM} / \mathrm{cr}$ \\
\hline Hyophila involuta (Hook.) A. Jaeger & $\mathrm{LM}, \mathrm{PM} / \mathrm{cr}, \mathrm{f}$ \\
\hline Hyophiladelphus agrarius (Hedw.) R.H. Zander & $\mathrm{LM}, \mathrm{PM} / \mathrm{ca}$ \\
\hline Hypopterygium tamarisci (Sw.) Brid. ex Müll. Hal. & LM \\
\hline Isopterygium byssobolax (Müll. Hal.) Paris & $\mathrm{UM} / \mathrm{f}$ \\
\hline Isopterygium jamaicense (E.B. Bartram) W.R. Buck & $\mathrm{UM}, \mathrm{LM}, \mathrm{PM} / \mathrm{cr}, \mathrm{f}$ \\
\hline Isopterygium subbrevisetum (Hampe) Broth. & LM/- \\
\hline Isopterygium tenerifolium Mitt. & $\mathrm{UM}, \mathrm{LM}, \mathrm{PM} / \mathrm{cr}, \mathrm{f}$ \\
\hline Isopterygium tenerum (Sw.) Mitt. & $\mathrm{UM}, \mathrm{LM}, \mathrm{PM} / \mathrm{cr}, \mathrm{f}$ \\
\hline Jaegerina scariosa (Lorentz) Arz. & $\mathrm{LM} / \mathrm{f}$ \\
\hline Jamesoniella rubricaulis (Nees) Grolle & $\mathrm{UM} / \mathrm{cr}$ \\
\hline Jungermannia sphaerocarpa Hook. & $\mathrm{UM} / \mathrm{cr}, \mathrm{f}$ \\
\hline Kurzia brasiliensis (Steph.) Grolle & $\mathrm{UM}, \mathrm{LM} / \mathrm{cr}, \mathrm{f}$ \\
\hline Kurzia capillaris (Sw.) Grolle & $\mathrm{UM}, \mathrm{LM}, \mathrm{PM} / \mathrm{cr}, \mathrm{f}$ \\
\hline Lejeunea caespitosa Lindenb. \& G.L.Nees & $\mathrm{LM} / \mathrm{f}$ \\
\hline Lejeunea cerina (Lehm. \& Lindenb.) Gottsche, Lindenb. \& Nees & $\mathrm{UM} / \mathrm{f}$ \\
\hline Lejeunea cochleata Spruce & $\mathrm{UM}, \mathrm{LM} / \mathrm{f}$ \\
\hline Lejeunea flava (Sw.) Nees & $\mathrm{UM}, \mathrm{LM} / \mathrm{cr}, \mathrm{f}$ \\
\hline Lejeunea grossitexta (Steph.) E. Reiner \& Goda & $\mathrm{UM} / \mathrm{f}$ \\
\hline Lejeunea immersa Spruce & $\mathrm{UM} / \mathrm{f}$ \\
\hline Lejeunea laetevirens Nees \& Mont. & $\mathrm{UM}, \mathrm{LM} / \mathrm{f}$ \\
\hline Lejeunea maxonii (Evans) X.-L. He & $\mathrm{LM}, \mathrm{PM} / \mathrm{f}$ \\
\hline Lejeunea oligoclada Spruce & $-/ f$ \\
\hline
\end{tabular}


Table 1. Continuation.

\begin{tabular}{|c|c|}
\hline Species & Elevational zone/phytophysiognomy \\
\hline Lejeunea phyllobola Nees \& Mont. ex Mont. & $\mathrm{UM} / \mathrm{f}$ \\
\hline Lejeunea raddiana Lindenb. & $\mathrm{UM} / \mathrm{f}$ \\
\hline Lepidolejeunea involuta (Gottsche) Grolle & $\mathrm{LM} / \mathrm{f}$ \\
\hline Lepidopilidium portoricense (Müll. Hal.) H.A. Crum & $\mathrm{LM} / \mathrm{f}$ \\
\hline Lepidopilum scabrisetum (Schwägr.) Steere & $\mathrm{LM} / \mathrm{f}$ \\
\hline Lepidozia coilophylla Taylor & $\mathrm{UM} / \mathrm{f}$ \\
\hline Lepidozia cupressina (Sw.) Lindenb. & $\mathrm{UM}, \mathrm{LM} / \mathrm{cr}, \mathrm{f}$ \\
\hline Lepidozia inaequalis (Lehm. \& Lindenb.) Gott. et. al. & $\mathrm{UM} / \mathrm{cr}, \mathrm{f}$ \\
\hline Leptodontium viticulosoides var. sulphureum (Müll. Hal.) R.H. Zander & $\mathrm{UM} / \mathrm{cr}, \mathrm{f}$ \\
\hline Leptoscyphus amphibolius (Nees) Grolle & $\mathrm{UM} / \mathrm{cr}$ \\
\hline Leucobryum albicans (Schwägr.) Lindb. & $\mathrm{UM}, \mathrm{LM} / \mathrm{cr}, \mathrm{f}$ \\
\hline Leucobryum albidum (Brid. ex P. Beauv.) Lindb. & $\mathrm{UM} / \mathrm{cr}$ \\
\hline Leucobryum clavatum Hampe & $\mathrm{UM} / \mathrm{f}$ \\
\hline Leucobryum clavatum var. brevifolium Broth. & $\mathrm{UM} / \mathrm{cr}$ \\
\hline Leucobryum crispum Müll. Hal. & $\mathrm{UM}, \mathrm{LM} / \mathrm{cr}, \mathrm{f}$ \\
\hline Leucobryum giganteum Müll. Hal. & $\mathrm{UM}, \mathrm{LM} / \mathrm{cr}, \mathrm{f}$ \\
\hline Leucobryum martianum (Hornsch.) Hampe ex Müll. Hal. & $\mathrm{UM}, \mathrm{LM}, \mathrm{PM} / \mathrm{cr}, \mathrm{f}$ \\
\hline Leucobryum sordidum Ångstr. & $\mathrm{LM} / \mathrm{cr}$ \\
\hline Leucolejeunea caducifolia Gradst. \& Schaeff.-Verwimp & $\mathrm{LM} / \mathrm{f}$ \\
\hline Leucolejeunea conchifolia (Evans) Evans & $\mathrm{LM} / \mathrm{f}$ \\
\hline Leucoloma cruegerianum (Müll. Hal.) A. Jaeger & $\mathrm{UM}, \mathrm{LM} / \mathrm{f}$ \\
\hline Leucoloma serrulatum Brid. & $\mathrm{UM}, \mathrm{LM} / \mathrm{cr}, \mathrm{f}$ \\
\hline Macrocoma brasiliensis (Mitt.) Vitt & $\mathrm{LM} / \mathrm{cr}$ \\
\hline Macrocoma cf. gastonyi D.H. Norris \& Vitt & $\mathrm{UM} / \mathrm{f}$ \\
\hline Macrocoma orthotrichoides (Raddi) Wijk \& Margad. & $\mathrm{LM}, \mathrm{PM} / \mathrm{f}$ \\
\hline Macrocoma tenuis subsp. sulivantii (Müll. Hal.) Vitt. & $\mathrm{LM} / \mathrm{f}$, ce \\
\hline Macromitrium cf. longifolium (Hook.) Brid. & $\mathrm{UM} / \mathrm{f}$ \\
\hline Macromitrium cirrosum (Hedw.) Brid. & $\mathrm{UM}, \mathrm{LM} / \mathrm{cr}, \mathrm{f}$ \\
\hline Macromitrium frustratum B.H. Allen & $\mathrm{UM} / \mathrm{f}$ \\
\hline Macromitrium microstomum (Hook. \& Grev.) Schwägr. & $\mathrm{UM}, \mathrm{LM} / \mathrm{cr}, \mathrm{f}$ \\
\hline Macromitrium podocarpi Müll. Hal. & $\mathrm{UM} / \mathrm{cr}, \mathrm{f}$ \\
\hline Macromitrium punctatum (Hook. \& Grev.) Brid. & $\mathrm{UM}, \mathrm{LM} / \mathrm{cr}, \mathrm{f}$ \\
\hline Macromitrium richardii Schwägr. & $\mathrm{LM} / \mathrm{cr}, \mathrm{f}$ \\
\hline Macromitrium sejunctum B.H. Allen & $\mathrm{UM} / \mathrm{f}$ \\
\hline Marchesinia brachiata (Sw.) Schiffn. & $\mathrm{UM}, \mathrm{LM} / \mathrm{cr}, \mathrm{f}$ \\
\hline Mastigolejeunea auriculata (Wilson \& Hook.) Schiffn. & $\mathrm{LM} / \mathrm{f}$ \\
\hline Mastigolejeunea plicatiflora (Spruce) Steph. & $\mathrm{LM} / \mathrm{f}$ \\
\hline Metalejeunea cucullata (Reinw., Blume \& Nees) Grolle & $\mathrm{UM} / \mathrm{f}$ \\
\hline Meteoridium remotifolium (Müll. Hal.) Manuel & $\mathrm{LM}, \mathrm{UM} / \mathrm{f}$ \\
\hline Meteorium nigrescens (Sw. ex Hedw.) Dozy \& Molk. & $\mathrm{LM} / \mathrm{f}$ \\
\hline Metzgeria brasiliensis Schiffn. & $\mathrm{LM} / \mathrm{f}$ \\
\hline Metzgeria cf. liebmanniana Lindenb. \& Gottsche & $\mathrm{UM} / \mathrm{f}$ \\
\hline Metzgeria decipiens (C. Massal.) Schiffn. & $\mathrm{UM} / \mathrm{f}$ \\
\hline Metzgeria furcata (L.) Dum & $\mathrm{LM} / \mathrm{f}$ \\
\hline
\end{tabular}


Table 1. Continuation.

\begin{tabular}{|c|c|}
\hline Species & Elevational zone/phytophysiognomy \\
\hline Metzgeria hegewaldii Kuwah. & $\mathrm{UM}, \mathrm{LM} / \mathrm{f}$ \\
\hline Metzgeria myriopoda Lindb. & $\mathrm{UM} / \mathrm{f}$ \\
\hline Metzgeria scyphigera A. Evans & $\mathrm{LM} / \mathrm{f}$ \\
\hline Microlejeunea bullata (Taylor) Steph. & $\mathrm{UM} / \mathrm{f}$ \\
\hline Microlejeunea cystifera Herzog & $\mathrm{LM} / \mathrm{f}$ \\
\hline Microlejeunea epiphylla Bischl. & $\mathrm{UM}, \mathrm{LM} / \mathrm{f}$ \\
\hline Micropterygium campanense Spruce ex Reimers & $\mathrm{LM} / \mathrm{f}$ \\
\hline Micropterygium reimersianum Herzog & $\mathrm{UM}, \mathrm{LM} / \mathrm{cr}, \mathrm{f}$ \\
\hline Micropterygium trachyphyllum Reimers & $\mathrm{UM} / \mathrm{cr}$ \\
\hline Mittenothaminium reptans (Hedw.) Cardot & $\mathrm{UM}, \mathrm{LM} / \mathrm{f}$ \\
\hline Mittenothamnium substriatum (Mitt.) Cardot & $\mathrm{UM} / \mathrm{f}$ \\
\hline Neckeropsis undulata (Hedw.) Reichardt & $\mathrm{LM} / \mathrm{f}$ \\
\hline Neesioscyphus homophyllus (Nees) Grolle & $\mathrm{UM} / \mathrm{f}$ \\
\hline Neesioscyphus sp. & $\mathrm{UM}$ \\
\hline Neurolejeunea breutelii (Gott.) A.Evans & $\mathrm{UM} / \mathrm{cr}, \mathrm{f}$ \\
\hline Nowellia curvifolia (Dicks.) Mitt. & $\mathrm{UM} / \mathrm{cr}$ \\
\hline Ochrobryum gardneri (Müll. Hal.) Mitt. & $\mathrm{LM} / \mathrm{cr}, \mathrm{f}$ \\
\hline Octoblepharum albidum Hedw. & $\mathrm{UM}, \mathrm{LM}, \mathrm{PM} / \mathrm{cr}, \mathrm{f}, \mathrm{ce}, \mathrm{ca}$ \\
\hline Octoblepharum cocuiense Mitt. & $\mathrm{UM}, \mathrm{LM}, \mathrm{PM} / \mathrm{cr}$ \\
\hline Octoblepharum cylindricum Schimp. ex Mont. & $\mathrm{LM} / \mathrm{cr}$ \\
\hline Octoblepharum erectifolium Mitt. ex R.S. Williams & $\mathrm{LM} / \mathrm{cr}, \mathrm{f}$ \\
\hline Octoblepharum pulvinatum (Dozy \& Molk.) Mitt. & $-/ \mathrm{cr}$ \\
\hline Odontolejeunea lunulata (F. Weber) Schiffn. & $-/ f$ \\
\hline Odontoschisma brasiliense Steph. & $\mathrm{UM} / \mathrm{cr}$ \\
\hline Odontoschisma denudatum (Nees) Dumort. & $\mathrm{UM}, \mathrm{LM} / \mathrm{cr}$ \\
\hline Odontoschisma falcifolium Steph. & $\mathrm{UM} / \mathrm{cr}$ \\
\hline Odontoschisma longiflorum Steph. & $\mathrm{LM} / \mathrm{cr}$ \\
\hline Omphalanthus filiformis (Sw.) Nees & $\mathrm{UM} / \mathrm{cr}, \mathrm{f}$ \\
\hline Orthodotium gracile (Wilson) Schwägr. ex B.S.G. & $\mathrm{LM} / \mathrm{f}$ \\
\hline Orthostichella versicolor (Müll. Hal.) B.H. Allen \& W.R. Buck & $\mathrm{LM} / \mathrm{cr}$ \\
\hline Orthostichopsis crinita (Sull.) Broth. & $\mathrm{UM} /-$ \\
\hline Orthostichopsis praetermissa W.R. Buck & $\mathrm{UM}, \mathrm{LM} / \mathrm{cr}, \mathrm{f}$ \\
\hline Orthostichopsis tetragona (Sw. ex Hedw.) Broth. & $\mathrm{LM} / \mathrm{f}$ \\
\hline Orthostichopsis tortipilis (Müll. Hal.) Broth. & $\mathrm{LM} / \mathrm{cr}, \mathrm{f}$ \\
\hline Oryzolejeunea saccatiloba (Steph.) Gradst. & $-/ f$ \\
\hline Pallavicinia lyellii (Hook.) Gray & $\mathrm{UM}, \mathrm{LM}, \mathrm{PM} / \mathrm{cr}, \mathrm{f}$ \\
\hline Philonotis cernua (Wilson) D.G. Griffin \& W.R. Buck & $\mathrm{UM} / \mathrm{cr}, \mathrm{f}$ \\
\hline Philonotis elongata (Dism.) H.A. Crum \& Steere & $\mathrm{LM} / \mathrm{f}$ \\
\hline Philonotis hastata (Duby) Wijk \& Margad. & $\mathrm{LM}, \mathrm{UM} / \mathrm{cr}, \mathrm{f}$ \\
\hline Philonotis sphaerocarpa (Hedw.) Brid. & $-/ \mathrm{cr}$ \\
\hline Philonotis uncinata (Schwägr.) Brid. & $\mathrm{PM} / \mathrm{cr}$ \\
\hline Phyllodon truncatulus (Müll. Hal.) W.R. Buck & $\mathrm{LM} / \mathrm{cr}$ \\
\hline Phyllogonium fulgens (Hedw.) Brid. & $\mathrm{LM} / \mathrm{f}$ \\
\hline Phyllogonium viride Brid. & $\mathrm{UM}, \mathrm{LM} / \mathrm{cr}, \mathrm{f}$ \\
\hline
\end{tabular}


Table 1. Continuation.

\begin{tabular}{|c|c|}
\hline Species & Elevational zone/phytophysiognomy \\
\hline Pilopogon guadalupensis (Brid.) J.-P. Frahm & LM \\
\hline Pilotrichella flexilis (Hedw.) Ångstr. & $\mathrm{UM} / \mathrm{f}$ \\
\hline Plagiochila aerea Taylor & $\mathrm{LM} / \mathrm{f}$ \\
\hline Plagiochila bifaria (Sw.) Lindenb. & $\mathrm{UM} / \mathrm{f}$ \\
\hline Plagiochila bryopterioides Spruce & $\mathrm{UM} / \mathrm{f}$ \\
\hline Plagiochila compressula (Nees) Lindenb. & $\mathrm{LM} / \mathrm{f}$ \\
\hline Plagiochila corrugata (Nees) Nees \& Mont. & $\mathrm{UM}, \mathrm{LM}, \mathrm{PM} / \mathrm{cr}, \mathrm{f}$ \\
\hline Plagiochila cristata (Sw.) Dumort. & $\mathrm{UM} / \mathrm{f}$ \\
\hline Plagiochila disticha (Lehm. \& Lindenb.) Mont. & $\mathrm{UM}, \mathrm{LM} / \mathrm{f}$ \\
\hline Plagiochila exigua (Taylor) Taylor & $\mathrm{UM} / \mathrm{f}$ \\
\hline Plagiochila fragilis Taylor & $\mathrm{UM} / \mathrm{cr}, \mathrm{f}$ \\
\hline Plagiochila gymnocalycina Lindenb. & $\mathrm{UM}, \mathrm{LM} / \mathrm{f}$ \\
\hline Plagiochila patentissima Steph. & $\mathrm{LM} / \mathrm{f}$ \\
\hline Plagiochila patula (Sw.) Lindenb. & $\mathrm{UM}, \mathrm{LM} / \mathrm{f}$ \\
\hline Plagiochila raddiana Lindenb & $\mathrm{LM} / \mathrm{f}$ \\
\hline Plagiochila rutilans Lindenb. & $\mathrm{UM} / \mathrm{cr}, \mathrm{f}$ \\
\hline Plagiochila simplex (Sw.) Lindenb. & $\mathrm{UM}, \mathrm{LM} / \mathrm{cr}, \mathrm{f}$ \\
\hline Plagiochila subplana Lindenb. & $\mathrm{UM} / \mathrm{f}$ \\
\hline Plaubelia sprengelii (Schwägr.) R.H. Zander & $\mathrm{LM}, \mathrm{PM} / \mathrm{ca}$ \\
\hline Pogonatum pensilvanicum (Hedw.) P. Beauv. & $\mathrm{UM} / \mathrm{f}$ \\
\hline Pohlia papillosa (Müll. Hal. ex A. Jaeger) Broth. & LM/- \\
\hline Polytrichum angustifolium Mitt. & $\mathrm{UM} / \mathrm{cr}, \mathrm{f}$ \\
\hline Polytrichum commune Hedw. & $-/ \mathrm{cr}$ \\
\hline Polytrichum juniperinum Hedw. & $\mathrm{UM}, \mathrm{LM} / \mathrm{cr}, \mathrm{f}$ \\
\hline Porella cf. reflexa (Lehm. \& Lindenb.) Trevis. & $\mathrm{LM} / \mathrm{f}$ \\
\hline Porella brasiliensis (Raddi) Schiffn. & $\mathrm{UM}, \mathrm{LM} / \mathrm{f}$ \\
\hline Porella swartziana (Weber) Trevis. & $\mathrm{LM} / \mathrm{f}$ \\
\hline Porothamnium leucocaulon (Müll. Hal.) M. Fleisch. & $\mathrm{UM} /-$ \\
\hline Pterogonidium pulchellum (Hook.) Müll. Hal. & $\mathrm{UM}, \mathrm{LM} / \mathrm{f}$ \\
\hline Pyrrhobryum spiniforme (Hedw.) Mitt. & $\mathrm{UM}, \mathrm{LM} / \mathrm{cr}, \mathrm{f}$ \\
\hline Racopilum tomentosum (Hedw.) Brid. & $\mathrm{LM} / \mathrm{cr}, \mathrm{f}$ \\
\hline Radula aff. conferta Lindenb. \& Gottsche & $\mathrm{UM} / \mathrm{f}$ \\
\hline Radula cubensis K. Yamada & $\mathrm{UM} / \mathrm{f}$ \\
\hline Radula fendleri Gottsche ex Steph. & $\mathrm{UM} / \mathrm{f}$ \\
\hline Radula inflexa Gottsche ex Steph. & $\mathrm{UM} / \mathrm{f}$ \\
\hline Radula javanica Gottsche & $\mathrm{UM} / \mathrm{cr}, \mathrm{f}$ \\
\hline Radula kegelii Gottsche & $\mathrm{UM}, \mathrm{LM} / \mathrm{f}$ \\
\hline Radula mexicana Steph. & $\mathrm{UM} / \mathrm{cr}, \mathrm{f}$ \\
\hline Radula pseudostachya Spruce & $\mathrm{UM} / \mathrm{f}$ \\
\hline Radula recubans Taylor & $\mathrm{UM} / \mathrm{f}$ \\
\hline Radula sinuata Steph. & $\mathrm{UM} / \mathrm{f}$ \\
\hline Radula tenera Mitt. ex Steph. & $\mathrm{LM} / \mathrm{f}$ \\
\hline Radula wrightii Castle & $\mathrm{UM} / \mathrm{f}$ \\
\hline Rhacopilopsis trinitensis (Müll. Hal.) E. Britton \& Dixon & $\mathrm{UM}, \mathrm{LM} / \mathrm{cr}$ \\
\hline
\end{tabular}


Table 1. Continuation.

\begin{tabular}{|c|c|}
\hline Species & Elevational zone/phytophysiognomy \\
\hline Rhacorcarpus purpurascens (Brid.) Par. & $\mathrm{UM} / \mathrm{cr}$ \\
\hline Rhodobryum aubertii (Schwägr.) Thér. & $\mathrm{LM} / \mathrm{f}$ \\
\hline Rhodobryum beyrichianum (Hornsch.) Müll. Hal. & $\mathrm{LM} / \mathrm{cr}, \mathrm{f}$ \\
\hline Rhodobryum grandifolium (Taylor) Schimp. & $-/ \mathrm{cr}, \mathrm{f}$ \\
\hline Rhodobryum roseum (Hedw.) Limpr. & $\mathrm{LM} / \mathrm{f}$ \\
\hline Riccardia cataractarum (Spruce) Schiffn. & $\mathrm{PM} / \mathrm{cr}$ \\
\hline Riccardia chamedryfolia (With.) Grolle & $\mathrm{LM} /-$ \\
\hline Riccardia digitiloba (Spruce ex Steph.) Pagán & $-/ \mathrm{ca}$ \\
\hline Riccia erythrocarpa Jovet-Ast. & $-/ \mathrm{ca}$ \\
\hline Riccia lindmanii Steph. & $\mathrm{LM} / \mathrm{ca}$ \\
\hline Riccia squamata Nees & $\mathrm{LM} / \mathrm{ca}$ \\
\hline Riccia vitalii Jovet-Ast. & $\mathrm{LM} / \mathrm{ca}$ \\
\hline Riccia weinionis Steph. & $\mathrm{LM} / \mathrm{ca}$ \\
\hline Rosulabryum billarderi (Schwägr.) J.R. Spence & $-/ \mathrm{cr}$ \\
\hline Rosulabryum capillare (Hedw.) J.R. Spence & $\mathrm{LM} / \mathrm{ce}$ \\
\hline Rosulabryum densifolium (Brid.) Ochyra & $\mathrm{UM}, \mathrm{LM} / \mathrm{f}$ \\
\hline Rosulabryum huillense (Welw. \& Duby) Ochyra & $\mathrm{LM} / \mathrm{f}$ \\
\hline Saccogynidium caldense (Ångstr.) Grolle & $\mathrm{UM} / \mathrm{f}$ \\
\hline Schiffneriolejeunea polycarpa (Nees) Gradst & $\mathrm{LM} / \mathrm{f}$ \\
\hline Schlotheimia jamesonii (Arn.) Brid. & $\mathrm{UM}, \mathrm{LM} / \mathrm{cr}, \mathrm{f}$ \\
\hline Schlotheimia rugifolia (Hook.) Schwägr. & $\mathrm{UM}, \mathrm{LM} / \mathrm{cr}, \mathrm{f}, \mathrm{ce}$ \\
\hline Schlotheimia tecta Hook. f. \& Wilson & $\mathrm{UM}, \mathrm{LM} / \mathrm{cr}, \mathrm{f}$ \\
\hline Schlotheimia torquata (Sw. ex Hedw.) Brid. & $\mathrm{UM}, \mathrm{LM} / \mathrm{cr}, \mathrm{f}$ \\
\hline Schlotheimia trichomitria Schwägr. & $\mathrm{UM}, \mathrm{LM} / \mathrm{cr}, \mathrm{f}$ \\
\hline Sematophyllum adnatum (Michx.) E. Britton & $\mathrm{UM}, \mathrm{LM} / \mathrm{cr}, \mathrm{f}, \mathrm{ce}, \mathrm{ca}$ \\
\hline Sematophyllum galipense (Müll. Hal.) Mitt. & $\mathrm{UM}, \mathrm{LM} / \mathrm{cr}, \mathrm{f}, \mathrm{ce}$ \\
\hline Sematophyllum sp. & UM, LM \\
\hline Sematophyllum subpinnatum (Brid.) E. Britton & $\mathrm{UM}, \mathrm{LM}, \mathrm{PM} / \mathrm{cr}, \mathrm{f}, \mathrm{ce}$ \\
\hline Sematophyllum subsimplex (Hedw.) Mitt. & $\mathrm{LM} / \mathrm{cr}, \mathrm{f}$ \\
\hline Sematophyllum swartzii (Schwägr.) W.H. Welch \& H.A. Crum & $\mathrm{UM}, \mathrm{LM} / \mathrm{cr}, \mathrm{f}$ \\
\hline Sematophyllum tequendamense (Hampe) Mitt. & $\mathrm{LM} / \mathrm{cr}, \mathrm{f}, \mathrm{ce}$ \\
\hline Sphagnum aciphyllum Müll. Hal. & UM,LM/- \\
\hline Sphagnum alegrense Warnst. & $\mathrm{UM} / \mathrm{f}$ \\
\hline Sphagnum brevirameum Hampe & $\mathrm{UM} / \mathrm{cr}$ \\
\hline Sphagnum capillifolium (Ehrh.) Hedw. & $\mathrm{UM}, \mathrm{LM} / \mathrm{cr}, \mathrm{f}$ \\
\hline Sphagnum chi-chiense H.A. Crum & $\mathrm{UM} / \mathrm{cr}$ \\
\hline Sphagnum contortulum H.A. Crum & $\mathrm{LM}, \mathrm{PM} / \mathrm{cr}$ \\
\hline Sphagnum harleyi H.A. Crum & $\mathrm{UM}, \mathrm{LM} / \mathrm{cr}$ \\
\hline Sphagnum longistolo Müll. Hal. & $\mathrm{UM}, \mathrm{LM}, \mathrm{PM} / \mathrm{cr}$ \\
\hline Sphagnum magellanicum Brid. & $\mathrm{UM}, \mathrm{LM} / \mathrm{cr}, \mathrm{f}$ \\
\hline Sphagnum oxyphyllum Warnst. & $\mathrm{UM}, \mathrm{LM} / \mathrm{cr}$ \\
\hline Sphagnum palustre $\mathrm{L}$. & $\mathrm{UM}, \mathrm{LM}, \mathrm{PM} / \mathrm{cr}, \mathrm{f}$ \\
\hline Sphagnum papillosum Lindb. & $-/ \mathrm{cr}$ \\
\hline
\end{tabular}


Table 1. Continuation.

\begin{tabular}{|c|c|}
\hline Species & Elevational zone/phytophysiognomy \\
\hline Sphagnum perichaetiale Hampe & $\mathrm{UM}, \mathrm{LM} / \mathrm{cr}, \mathrm{f}$ \\
\hline Sphagnum recurvum P. Beauv. & $\mathrm{UM}, \mathrm{LM} / \mathrm{cr}, \mathrm{f}$ \\
\hline Sphagnum sparsum Hampe & $\mathrm{LM} / \mathrm{cr}$ \\
\hline Sphagnum strictum Sull. & $-/ \mathrm{cr}$ \\
\hline Sphagnum subsecundum Nees & $\mathrm{UM}, \mathrm{LM} / \mathrm{cr}, \mathrm{f}$ \\
\hline Sphagnum vitalii H.A. Crum & $\mathrm{UM}, \mathrm{LM} / \mathrm{f}$ \\
\hline Squamidium brasiliense (Hornsch.) Broth. & $\mathrm{UM}, \mathrm{LM} / \mathrm{f}$ \\
\hline Squamidium leucotrichum (Taylor) Broth. & $\mathrm{LM} / \mathrm{f}$ \\
\hline Squamidium nigricans (Hook.) Broth. & $\mathrm{LM} / \mathrm{f}$ \\
\hline Symphyogyna aspera Steph. ex MacCormick & $\mathrm{LM} / \mathrm{cr}$ \\
\hline Symphyogyna brasiliensis Nees & $\mathrm{LM} / \mathrm{cr}$ \\
\hline Symphyogyna leptothelia Taylor & $\mathrm{PM} / \mathrm{cr}$ \\
\hline Symphyogyna podophylla (Thunb.) Mont. \& Nees & $\mathrm{UM} / \mathrm{cr}$ \\
\hline Syrrhopodon elongatus Sull. & $\mathrm{UM} / \mathrm{f}$ \\
\hline Syrrhopodon elongatus var. glaziovii (Hampe) W.D. Reese & $\mathrm{LM} / \mathrm{cr}, \mathrm{f}$ \\
\hline Syrrhopodon gardneri (Hook.) Schwägr. & $\mathrm{UM}, \mathrm{LM} / \mathrm{cr}, \mathrm{f}$ \\
\hline Syrrhopodon gaudichaudii Mont. & $\mathrm{UM}, \mathrm{LM} / \mathrm{cr}, \mathrm{f}$ \\
\hline Syrrhopodon incompletus Schwägr. & $\mathrm{LM} /-$ \\
\hline Syrrhopodon leprieurii Mont. & $\mathrm{LM} / \mathrm{cr}$ \\
\hline Syrrhopodon ligulatus Mont. & $\mathrm{LM} / \mathrm{cr}, \mathrm{f}$ \\
\hline Syrrhopodon lycopodioides (Sw. ex Brid.) Müll. Hal. & $-/ \mathrm{cr}$ \\
\hline Syrrhopodon parasiticus (Sw. ex Brid.) Paris & $\mathrm{UM}, \mathrm{LM}, \mathrm{PM} / \mathrm{f}$ \\
\hline Syrrhopodon prolifer Schwägr. & $\mathrm{UM}, \mathrm{LM}, \mathrm{PM} / \mathrm{cr}, \mathrm{f}$ \\
\hline Syrrhopodon prolifer var. acanthoneuros (Müll. Hal.) Müll. Hal. & $\mathrm{LM} /-$ \\
\hline Syrrhopodon prolifer var. scaber (Mitt.) W.D. Reese & $\mathrm{LM} / \mathrm{cr}, \mathrm{f}$ \\
\hline Syrrhopodon prolifer var. tenuifolius (Sull.) W.D. Reese & $\mathrm{UM} / \mathrm{cr}$ \\
\hline Syzygiella aff. integerrima Steph. & $\mathrm{UM} / \mathrm{f}$ \\
\hline Syzygiella liberata Inoue & $\mathrm{UM} / \mathrm{cr}, \mathrm{f}$ \\
\hline Taxiphyllum taxirameum (Mitt.) M. Fleisch. & $\mathrm{LM} /-$ \\
\hline Taxithelium planum (Brid.) Mitt. & $-/ f$ \\
\hline Telaranea diacantha (Mont.) J.J. Engel \& G.L. Merr. & $\mathrm{UM}, \mathrm{LM} / \mathrm{f}$ \\
\hline Telaranea nematodes (Gott. ex Aust.) Howe & $\mathrm{UM}, \mathrm{LM}, \mathrm{PM} / \mathrm{cr}, \mathrm{f}$ \\
\hline Thamniopsis undata (Hedw.) W.R. Buck & $\mathrm{LM} / \mathrm{cr}$ \\
\hline Thuidium delicatulum (Hedw.) Schimp. & $\mathrm{UM} / \mathrm{f}$ \\
\hline Thuidium subtamariscinum (Hampe) Broth. & $-/ \mathrm{cr}$ \\
\hline Thuidium tomentosum Schimp. & $\mathrm{LM} / \mathrm{f}$ \\
\hline Thuidium urceolatum Lorentz & $\mathrm{LM} / \mathrm{f}$ \\
\hline Tortella humilis (Hedw.) Jenn. & $\mathrm{UM}, \mathrm{LM}, \mathrm{PM} / \mathrm{cr}, \mathrm{f}$ \\
\hline Tortella tortuosa (Hedw.) Limpr. & $\mathrm{UM} / \mathrm{f}$ \\
\hline Trichocolea brevifissa Steph. & $\mathrm{UM} / \mathrm{f}$ \\
\hline Trichocolea flaccida (Spruce) Jack \& Steph. & $\mathrm{UM} / \mathrm{cr}$ \\
\hline Trichosteleum microstegium (Besch.) A. Jaeger & $\mathrm{LM} / \mathrm{cr}, \mathrm{f}$ \\
\hline
\end{tabular}


Table 1. Continuation.

\begin{tabular}{lc}
\hline Species & Elevational zone/phytophysiognomy \\
\hline Trichosteleum sentosum (Sull.) A. Jaeger & $\mathrm{LM} / \mathrm{cr}, \mathrm{f}$ \\
Trichosteleum subdemissum (Schimp. ex Besch.) A. Jaeger & $\mathrm{LM} / \mathrm{cr}$ \\
Trichostomum tenuirostre (Hook. \& Tayl.) Lindb. & $\mathrm{UM}, \mathrm{PM} / \mathrm{f}$ \\
Wijkia flagellifera (Broth.) H.A. Crum & $\mathrm{UM}, \mathrm{LM} / \mathrm{cr}, \mathrm{f}$ \\
Wijkia subnitida (Hampe) H.A. Crum & $\mathrm{UM} / \mathrm{f}$ \\
Zelometeorium patulum (Hedw.) Manuel & $\mathrm{LM} / \mathrm{f}$ \\
Zoopsidella integrifolia (Spruce) R. M. Schust. & $\mathrm{LM} / \mathrm{f}$ \\
\hline
\end{tabular}

ca - caatinga; c - cerrado; cr - campos rupestres; $\mathrm{f}$ - forest; - - not determined; UM - upper montane; LM - lower montane; PM - premontane.

cells and chlorophyll-containing cells directly exposed to the light. However, such species have a low chlorophyll $a / b$ ratio, are capable of adjusting their number of chloroplasts according to the levels of light and are poikilohydric, having the ability to rehydrate rapidly (Glime 2007).

In campos rupestres, the most representative families were Lepidoziaceae, Pallaviciniaceae, Jungermanniaceae, which also include members with high demand for shaded areas and humidity, as well as Sphagnaceae, Bartramiaceae, Calymperaceae and Leucobryaceae. The latter group includes species with morphological and physiological adaptations to high light intensity and desiccation and therefore have a better chance of enduring prolonged exposure to sunlight, such adaptations including the presence of hyaline cells, which filter the light reaching the photosynthesis cells; cancellinae, for water storage; and hydroids, cells that constitute the water conduction and support tissues (Glime 2007; Proctor 2007).

The particularities of the environmental conditions of the caatinga allow the occurrence of families such as Ricciaceae, which are adapted to the typical arid conditions of the region (Jovet-Ast 1991). Species of the genus Riccia, which are common residents of ephemeral habitats, absorb water by capillarity among the rhizoids on the inferior surface of the thallus. Under drought conditions, the gametophyte furls itself, exposing the rhizoids, which serve to absorb water and provide a reflective surface that protects the chlorophyll-containing cells of the thallus. Some species are capable of surviving for seven years in this desiccated state, and even if the vegetative part dies, the spores can persist because of the great quantity of nutrients they store. The annual species compensate for this water loss by producing a large number of spores and using their ornamentation to attract animals for their dispersion (Glime 2007; Vanderpoorten \& Goffinet 2009). As in the present study, other floristic studies of bryophytes in the caatinga (Pôrto et al. 1994; Bastos et al. 1998b) have shown low species richness, with representative species typically being tolerant of exposure to intense light and heat, such as those of the families Pottiaceae and Ricciaceae. A little over one third of the species recorded in the present study were also included in the flora listed in the literature cited above.
We found no families that were exclusive to the cerrado, and the flora of our study sites had low floristic affinity with those described in other studies of the cerrado in northeastern Brazil (Vilas Bôas-Bastos \& Bastos 1998; Castro et al. 2002). It is noteworthy that the areas of cerrado investigated in our study were quite dry, and some bore evidence of recent fires.

Among the bryophytes we identified in the Chapada Diamantina ecoregion, the geographic distribution in Brazil was categorized as restricted for $30 \%$ of the taxa, moderate for $32 \%$ and broad for $38 \%$. Many of the taxa classified as having a restricted or moderate distribution had previously been recorded only for the southeastern region of Brazil, particularly in mountainous areas, where environmental conditions, such as the mild temperatures and cloud cover associated with the upper elevations, enable the survival of species less tolerant to heat and desiccation. This corroborates the findings of Nascimento et al. (2010) regarding the floristic and biogeographical relationships between the arboreal flora of the upper montane forests in the south of the Chapada Diamantina ecoregion and those of the seasonal forest and rain forest formations in the Atlantic Forest of southeastern and southern Brazil, those authors having identified great floristic affinity between the two regions. In that same study, the authors point out a floristic continuation between the Serra do Espinhaço, to the south, and the central-south portion of the Chapada Diamantina ecoregion, as well as the occurrence of an extensive biogeographical province that connects the Chapada Diamantina ecoregion with the Serra da Mantiqueira, forming a corridor composed of campos rupestres and shrub-tree vegetation. That corridor would allow the transit of plant and animal species adapted to grassland and forest environments, where the humidity is higher and the temperatures are milder.

Our analysis of the worldwide geographical distribution of the bryophyte species identified showed a predominance of neotropical species (42\%), followed by disjunct species (12\%); cosmopolitan species (10\%); pantropical species (9\%); and species endemic to Brazil (4\%), to the American tropics (3\%) and to the American subtropics America (8\%). In the upper elevations of the Chapada Diamantina ecoregion, there was a significant number of disjunct species with the distribution pattern eastern Brazil-Andes region (14 species), as previously observed for mountainous regions 
in southeastern Brazil (Santos \& Costa 2010b), which can be explained by the similarities in climatic conditions between eastern Brazil and the Andes region. Such species, all of which were sampled in campos rupestres or forests, included Chiloscyphus latifolius (found in Brazil and Bolivia); Radula tenera, Sematophyllum tequendamense, Syrrhopodon helicophyllus and Syzygiella liberata (found in Brazil and Colombia); Radula sinuata (found in Brazil, Bolivia and Colombia); Metzgeria hegewaldii (found in Brazil and Peru); Micropterygium reimersianum (found in Brazil and Venezuela); Micropterygium campanense (found in Brazil, Venezuela and Peru); Lepidozia brasiliensis (found in Brazil, Colombia, Ecuador and Peru); Calypogeia andicola (found in Brazil, Colombia and Ecuador); Lepidozia inaequalis (found in Brazil, Ecuador, Bolivia and Peru); Plagiochila fragilis - (found in Brazil and Ecuador); and Drepanolejeunea campanulata (found in Brazil and the northern Andes). Other disjunctions were represented by Adelothecium bogotense (found throughout the neotropics, as well as in Madagascar and Tanzania); Aphanolejeunea asperrima (found in Brazil and Patagonia; Archidium clavatum (found in Brazil and Australia); Riccia vitalii (found in Brazil and Costa Rica); Radula cubensis and R. wrightii (found in Brazil and Cuba); Diplasiolejeunea latipuense (found in Brazil and Guyana); Microlejeunea cystifera (found in Brazil and French Guyana); Acroporium caespitosum and Wijkia flagellifera (found in Brazil and the West Indies); Neesioscyphus homophyllus (found in southeastern Brazil and northern Argentina); Eccremidium floridanum (found in Brazil and the United States); Daltonia pulvinata (found throughout the neotropics and on Reunion Island); Schlotheimia tecta (found throughout the neotropics and in India); Jamesoniella rubricaulis (found throughout the neotropics and in the Azores); and Bryum paradoxum (found throughout the neotropics and in Asia).

Four of the taxa identified are endemic to the Chapada Diamantina ecoregion, all of them belonging to the family Sphagnaceae and sampled in campos rupestres: Sphagnum chichiense var. uvidulum; S. harleyi; S. contortulum; and S. vitalii.

Bryophyte composition differed among the three elevational zones studied, species richness and the numbers of exclusive taxa being highest in the lower and upper montane formations: premontane (40 species, seven exclusive); lower montane (246 species, 121 exclusive), upper montane (241 species, 96 exclusive). Only eight taxa were sampled in all three zones; the premontane and lower montane zones had 33 taxa in common; and the lower and upper montane zones had 51 taxa in common.

The bryophyte flora found in the upper montane elevational zone of the Chapada Diamantina ecoregion comprise taxa previously identified as typical of mountainous regions in Brazil (Gradstein \& Costa 2003; Costa \& Lima 2005; Santos \& Costa 2010a): Adelanthus decipiens, Aptychopsis pyrrophylla, Atractylocarpus brasiliensis, Campylopus dichrostis, C. julicaulis, Drepanolejeunea araucariae, Frulania arecae, Herbertus juniperoideus ssp. bivittatus, Harpale- jeunea subacuta, Jamesoniella rubricaulis, Jungermannia sphaerocarpa, Microlejeunea cystifera, Neesioscyphus homophyllus, Odontoschisma denudatum, Plagiochila cristata, Polytrichum brasiliense, Rhacocarpus purpurascens, Radula fendleri, Saccogynidium caldense, Schloteimia trichomitria, Sphagnum alegrense, Syzygiella aff. integerrima, S. liberata and Trichocolea flaccida.

Among the species that occurred in the lower and upper montane formations and those of restricted distribution in Brazil, some are distinctive for their rarity within the Chapada Diamantina ecoregion, having been collected in forests or campos rupestres areas, with only one to five occurrences: Adelothecium bogotense, Anastrophyllum piligerum, Harpalejeunea subacuta, Jungermannia sphaerocarpa, Lophocolea mandonii, Micropterygium campanense, Neesioscyphus homophyllus, Odontoschisma brasiliense, Sematophyllum swartzii, Sphagnum alegrense and Syzygiella liberata. Some of those species have been listed as threatened in other Brazilian states.

Based on the results of this study, we conclude that the Chapada Diamantina ecoregion is an important center of bryophyte diversity, with high species richness, representing approximately $63 \%$ of the bryophyte flora of Bahia. A considerable proportion (83\%) of the species identified were exclusive to montane zones (lower and upper), $30 \%$ being exclusive to the upper montane zone, mainly distributed in montane forests and campos rupestres. Despite its geological, ecological and biological importance, as well as its considerable size, to date only nine conservation units have been established in the region, representing only $8.1 \%$ of its area, and only three of those are totally protected areas, corresponding to a mere $3.9 \%$ of its total area (MMA 2005). Therefore, we can highlight the importance of preserving this region and of reducing the destructive impact of human activity, not only in the three areas already recognized for their high levels of diversity (the Serra do Barbado Environmentally Protected Area; Sete Passagens State Park; and Chapada Diamantina National Park) but also in the campos rupestres, seasonal forests and rain forests of the surrounding areas such as the municipalities of Piatã and Morro do Chapéu.

\section{Acknowledgments}

This study received financial support from the Brazilian Conselho Nacional de Desenvolvimento Científico e Tecnológico (CNPq, National Council for Scientific and Technological Development; Doctoral Scholarship Grant to E.B.V.) and from the Fundação de Amparo à Pesquisa do Estado da Bahia (FAPESB, Foundation for the Support of Research in the State of Bahia; Grant no. APR 0066/2007). The authors are grateful to the Federal University of Feira de Santana Department of Biological Sciences for providing access to the infrastructure necessary for the execution of the study (Mycology Laboratory and Herbarium). 


\section{References}

Agritempo. 2010. Agritempo site. URL: http://www.agritempo.gov.br (Accesso em 05/2010).

Ah-Peng, C.; Chualh-Petiot, M.; Descamps-Julien, B.; Bardat, J.; Stamenoff, P. \& Strasberg, D. 2007. Bryophyte diversity and distribution along an altitudinal gradient on a lava flow in La reunion. Diversity and Distributions 13: 654-662.

Alvarenga, L.D.P. \& Pôrto, K.P. 2007. Patch size and isolation effects on epiphytic and epiphyllous bryophytes in the fragmented Brazilian Atlantic forest. Biological Conservation 134: 415-427.

Andrew, N.R.; Rodgerson, L. \& Dunlop, M. 2003. Variation in invertebrate-bryophyte community structure at different spatial scales along altitudinal gradients. Journal of Biogeography 30: 731-746.

Ballejos, J. \& Bastos, C.J.P. 2009a. Musgos Pleurocárpicos do Parque Estadual das Sete Passagens, Miguel Calmon, Bahia, Brasil. Hoehnea 36: 479-495.

Ballejos, J. \& Bastos, C.J.P., 2009b. Orthotrichaceae e Rhizogoniaceae (Bryophyta - Bryopsida) do Parque Estadual das Sete Passagens, Bahia, Brasil. Rodriguésia 60: 723-733.

Bastos C.J.P. \& Valente, E.B. 2008. Hepáticas (Marchantiophyta) da Reserva Ecológica da Michelin, Igrapiúna, Bahia, Brasil. Sitientibus - Série Ciências Biológicas 8: 280-293.

Bastos C.J.P. \& Vilas Bôas-Bastos, S.B. 2008. Musgos acrocárpicos e cladocárpicos (Bryophyta) da Reserva Ecológica da Michelin, Igrapiúna, Bahia, Brasil. Sitientibus - Série Ciências Biológicas 8: 275-279.

Bastos, C.J.P. \& Yano, O. 2009. O gênero Lejeunea Libert (Lejeuneaceae) no Estado da Bahia, Brasil. Hoehnea 36: 303-320.

Bastos C.J.P.; Stradmann, M.T. \& Vilas Bôas-Bastos, S.B. 1998a. Additional contribution to the bryophyte flora from Chapada Diamantina National Park, State of Bahia, Brazil. Tropical Bryology 15: 15-20.

Bastos, C.J.P.; Albertos, B. \& Vilas-Bôas, S.B. 1998b Bryophytes from some 'caatinga' areas in the state of Bahia (Brazil). Tropical Bryology 14: 69-75.

Bastos, C.J.P.; Yano, O \& Vilas Bôas-Bastos, S.B. 2000. Briófitas de campos rupestres da Chapada Diamantina, Estado da Bahia, Brasil. Revista Brasileira de Botânica 23: 357-368.

Brummitt, R.K. \& Powell, C.E. 1992. Authors of plant names. Royal Botanic Gardens, Kew.

Buck, W.R. 1998. Pleurocarpous Mosses of the West Indies. Memoirs of The New York Botanical Garden 1: 1-401.

Câmara, P.E.A.S. 2008a. Musgos Acrocárpicos da reserva Ecológica do IBGE, DF. Brasil. Acta Botanica Brasilica 22(4): 1027-1035.

Câmara, P.E.A.S. 2008b. Musgos pleurocárpicos das matas de galeria da Reserva Ecológica do IBGE, RECOR, Distrito Federal, Brasil. Acta Botanica Brasilica 22(2): 573-581.

Câmara, P.E.A.S. \& Costa, D.P. 2006. Hepáticas e antóceros das matas de galeria da Reserva Ecológica do IBGE, RECOR, Distrito Federal, Brasil. Hoehnea 33(1): 41-49.

Campelo, M.J.A. \& Pôrto, K.C. 2007. Brioflora epífita e epífila da RPPN Frei Caneca, Jaqueira, PE, Brasil. Acta Botanica Brasilica 21(1): 185-192.

Castro, N.M.C.F.; Porto, K.C.; Yano, O. \& Castro, A.A.J.F. 2002. Levantamento florístico de Bryopsida de cerrado e mata ripícola do Parque Nacional de Sete Cidades, Piauí, Brasil. Acta Botanica Brasilica 16(1): 61-76

Clarke, K.R. \& Warwick, R.M., 1994. Change in Marine Communities: An Approach to Statistical Analysis and Interpretation. Natural Enviroment Reseacrh Council, UK.

Conceição, A.A.; Rapini, A.; Pirani, J.R.; Giulietti, A.M; Harley, R.M.; Silva, T.R.; Santos, A.K.; Correia, C.; Andrade, I.M; Costa, J.A.S.; Souza, L.R.S.; Andrade, M.J.G; Funch, R.R.; Freitas, T.A.; Freitas, A.M.M. \& Oliveira, A.A. 2005. Campos Rupestres. Pp. 153-180. In: Juncá, F.A.; Funch, L. \& Rocha, W. (Eds.). Biodiversidade e Conservação da Chapada Diamantina. Brasília, Ministério do Meio Ambiente.

Costa D.P. 1999. Epiphytic Bryophyte Diversity in Primary and Secondary Lowland Rainforest in Southeastern Brazil. The Bryologist 102: 320-326.

Costa, D.P. \& Lima, F.M. 2005. Moss diversity in the tropical rainforest of Rio de Janeiro, Southeastern Brazil. Revista Brasileira de Botânica 28(4): 671-685
Costa, D.P. \& Silva, A.G. 2003. Briófitas da reserva Natural da Vale do Rio Doce, Linhares, Espírito Santo, Brasil. Boletim do Museu de Biologia Mello Leitão 16: 21-38

Crandall-Stotler. B.; Stotler R.E. \& Long, D.G. 2009. Morphology and classification of the Marchantiophyta. Pp. 1-54. In: Goffinet, B. \& Shaw, A.J. (Eds.). Bryophyte Biology. Second edition.

Crum, H. 1984. North American Flora, Series II. Sphagnopsida. Sphagnaceae. The New York Botanical Garden 11: 1-180.

Egunyomi, A. \& Vital, D.M. 1984. Comparative studies on the bryofloras of the nigerian savanna and the brazilian cerrado. Revista Brasileira de Botânica 7: 129-136.

Forzza, R.C.; Leitman, P.M.; Costa, A.F.; Carvalho Jr., A.A.; Peixoto, A.L.; Walter, B.M.T.; Bicudo, C.; Zappi, D.; Costa, D.P.; Lleras, E.; Martinelli, G.; Lima, H.C.; Prado, J.; Stehmann, J.R.; Baumgratz, J.F.A.; Pirani, J.R.; Sylvestre, L.; Maia, L.C.; Lohmann, L.G.; Queiroz, L.P.; Silveira, M.; Coelho, M.N.; Mamede, M.C.; Bastos, M.N.C.; Morim, M.P.; Barbosa, M..; Menezes, M.; Hopkins, M.; Secco, R.; Cavalcanti, T. B. \& Souza, V.C. 2012 Introdução. In: Lista de Espécies da Flora do Brasil. Jardim Botânico do Rio de Janeiro. Disponível em http://floradobrasil.jbrj.gov.br/2012/ (Acesso em 24/11/2012). Frahm, J-P. 1990. The ecology of epiphytic bryophytes on Mt. Kinabalu. Sabah (Malaysia). Nova Hedwigia 51: 121-132.

Frahm, J.-P. 1991. Dicranaceae: Campylopodioideae, Paraleucobryoideae. Flora Neotropica, monograph 54: 1-237.

Frahm, J.-P. 1994. The ecology of epiphytic bryophytes on Mt. Kahuzi (Zaire). Tropical Bryology 9: 137-151.

Frahm, J-P. \& Gradstein, S.R. 1991. An altitudinal zonation of tropical rain forests using bryophytes. Journal of Biogeography 18: 669-678.

Funch, L.S. 2008. Florestas do Parque Nacional da Chapada Diamantina e seu entorno. Pp. 63-77. In: Funch, L.S., Funch, R.R. \& Queiroz, L.P. (Orgs.) Serra do Sincorá: Parque Nacional da Chapada Diamantina. Radami Editora Gráfica, Feira de Santana.

Funch, L.S.; Funch, R.R.; Harley, R.; Giulietti, A.M.; Queiroz, L.P.; França F.; Melo, E.; Gonçalves, C.N. \& Santos, T. 2005. Florestas Estacionais Semideciduais. Pp. 181-193. In: Juncá, F.A.; Funch, L. \& Rocha, W. (Eds.). Biodiversidade e Conservação da Chapada Diamantina. Brasília, Ministério do Meio Ambiente.

Germano S.R. \& Pôrto K.C. 1996. Floristic survey of epixylic bryophytes of an area remnant of the Atlantic Forest (Timbaúba, PE, Brazil) 1. Hepaticopsida (except Lejeuneaceae) and Bryopsida. Tropical Bryology 12: 21-28.

Giulietti, A.M. \& Pirani, J.R. 1988. Patterns of geographic distribution of some plant species from the Espinhaço Range, Minas Gerais and Bahia, Brazil. Pp. 39-69. In: Vanzolini, P.E. \& Heyer, W.R. (Eds.). Proceedings of a workshop on Neotropical Distribution Patterns. Rio de Janeiro, Academia Brasileira de Ciências.

Giulietti, A.M.; Pirani, J.R. \& Harley, R.M. 1997. Espinhaço Range Region, Eastern Brazil. Pp. 397-404. In: S.D. Davis, V.H. Heywood, O. Herrera-Macbryde, J. Villa-Lobos \& A.C. Hamilton (Eds.). Centres of plant diversity. A guide and strategy for their conservation. v.3. The Americas. Cambridge, IUCN Publication Unity.

Glime, Janice M. 2007. Bryophyte Ecology. Volume 1. Physiological Ecology. Ebook sponsored by Michigan Technological University and the International Association of Bryologists. http://www.bryoecol.mtu. edu/. (Accesso em 01/2010).

Goffinet, B.; Buck, W.R. \& Shaw, A. J. 2009. Morphology, anatomy, and classification of the Bryophyta. Pp. 55-138. In: B. Goffinet \& A.J. Shaw (Eds.). Bryophyte Biology. Second edition. Cambridge, Cambridge University Press.

Gradstein, S.R. 1995. Bryophyte diversity of the tropical rainforest. Archives des Sciences [Société de physique et d'histoire naturelle de Genève] 48: 91-96.

Gradstein \& Costa, D.P. 2003. The Hepaticae and Anthocerotae of Brazil. Memoirs of The New York Botanical Garden 87: 1-336.

Gradstein, S.R. \& Pócs, T. 1989. Bryophytes. Pp. 311-325. In: H. Lieth \& M.J.A. Werger (Eds.). Tropical Rain Forest Ecosystems. Amsterdam, Elsevier Science Publischers B.V.

Gradstein, S.R.; Churchil, S.P. \& Salazar-Allen, N. 2001. Guide to the Bryophytes of Tropical America. Memoirs of The New York Botanical Garden 86: 1-577. 
Harley, R.M. 1995. Bryophyta. Pp. 803-812. In: Stannard, B.L. (Ed.). Flora of the Pico das Almas. Chapada Diamantina - Bahia, Brazil. Kew, Royal Botanic Garden.

Harley, R.M.; Giulietti, A.M.; Grilo, A.; Silva, T.S.; Funch, L.; Funch, R.R.; Queiroz, L.P.; França, F.; Melo, E.; Gonçalves, C.N. \& Nascimento, F.H.F. 2005. Cerrado. Pp. 121-152. In: Juncá, F.A.; Funch, L. \& Rocha, W. (Org.). Biodiversidade e Conservação da Chapada Diamantina. Brasília, Ministério do Meio Ambiente.

IBGE. 2004. Mapa de Biomas do Brasil. Ministério do Planejamento, Orçamento e Gestão.

Instituto de Pesquisas Jardim Botânico do Rio de Janeiro (Briófitas). 2008. http://www.jbrj.gov.br/pesquisa/div_tax/briofitas (Accesso em 01/2010)

Jovet-Ast, S. 1991. Riccia (Hépatique, Marchantiales) d’Amérique Latine, Taxons du sous-genre Riccia. Cryptogamie, Bryologie et Lichénologie 12: 189-370.

Kessler, M. 2000. Altitudinal zonation of Andean cryptogam communities. Journal of Biogeography 27: 275-282.

Mägdefrau, K. 1982. Life-forms of bryophytes. Pp. 45-58. In: Smith, A.J.E. (Ed.). Bryophyte Ecology. Chapman and Hall. Cambridge, Cambridge University Press.

MMA - Ministério do meio ambiente. 2005. Biodiversidade e conservação da Chapada Diamantina. Brasília: Ministério do Meio Ambiente.

Molinaro, L.C. \& Costa, D.P. 2001. Briófitas do arboreto do Jardim Botânico do Rio de Janeiro. Rodriguésia 81: 107-124.

Nascimento, F.H.F., Giulietti, A.M. \& Queiroz, L.P. 2010. Diversidade arbórea das florestas alto montanas no Sul da Chapada Diamantina, Bahia, Brasil. Acta Botanica Brasilica 24(3): 674-685.

Nolasco, M.C., Lima, C.C.U., Rocha, W.F. \& Rêgo, M.J.M. 2008. Aspectos físicos da Serra do Sincorá, Chapada Diamantina, Bahia. Pp. 17-33. In: Funch, L.S.; Funch, R.R. \& Queiroz, L.P. (Orgs.) Serra do Sincorá: Parque Nacional da Chapada Diamantina.

Oliveira-Filho, A.T.; Jarenkow, J.A. \& Rodal, M.J.N. 2006. Floristic relationships of seasonally dry forests of eastern South America based on tree species distribution patterns. Pp. 159-192. In: Pennington, R.T.; Ratter, J.A. \& Lewis, G.P. (Eds.) Neotropical savannas and dry forests: Plant diversity, biogeography and conservation. The Systematics Association Special volume Series 69, Florida, Boca Raton, CRC Press - Taylor and Francis Group.

Peralta, D.F. \& Vital, D.M. 2006. Archidiaceae (Archidiales, Bryophyta) do Brasil. Boletim do Instituto de Botânica 18: 17-32.

Peralta, D.F. \& Yano, O. 2005. Briófitas de mata paludosa, município de Zacarias, noroeste do Estado de São Paulo, Brasil. Acta Botanica Brasilica 19(4): 963-977.

Pócs, T. 1982. Tropical forest bryophytes. Pp. 59-104. In:. Smith, A.J.E (Ed.). Bryophyte Ecology. London, Chapman and Hall.

Pôrto K.C. 1990. Bryoflores d'une forêt de plaine et d'une forêt d'altitude moyenne dans l'État de Pernambuco (Brésil); Analyse floristique. Cryptogamie, Bryologie et Lichénologie 11: 109-161.

Pôrto K.C. 1992. Bryoflores d'une forêt de plaine et d'une forêt d'altitude moyenne dans l'État de Pernambuco (Brésil); Analyse écologique comparative des forêts. Cryptogamie, Bryologie et Lichénologie 13: $187-219$.

Pôrto, K.C.; Silveira M.F.G.; \& Sá, P.S. A. 1994. Briófitas da 'caatinga' 1. Estação experimental do IPA, Caruaru, PE. Acta Botanica Brasilica 8: 77-85.

Proctor, M.C.F.; Oliver, M. J.; Wood, A. J.; Alpert, P.; Stark, L.R.; Cleavitt, N. L. \& Mishler, B. D. 2007. Desiccation-tolerance in bryophytes: a review. The Bryologist 110: 595-621.

Pursell, R. A. 2007. Fissidentaceae. Flora Neotropica, Monograph 101: 1-279.

Queiroz, L.P.; França, F.; Giulietti, A.M.; Melo, E.; Gonçalves, C.N.; Funch, L.S.; Harley, R.M.; Funch, R.R. \& Silva, T.S. 2005. Caatinga. Pp. 95120. In: Juncá, F.A., Funch, L. \& Rocha, W. (Orgs.). Biodiversidade e Conservação da Chapada Diamantina. Ministério do Meio Ambiente.Queiroz, L.P.;

Funch, L.S. \& Funch, R.R. 2008. Vegetação da Chapada Diamantina-Ênfase no Parque Nacional da Chapada Diamantina. Pp. 35-42. In: Funch, L.S., Funch, R.R. \& Queiroz, L.P. (Orgs.) Serra do Sincorá: Parque Nacional da Chapada Diamantina.
Reese, W.D. 1993. Calymperaceae. Flora Neotropica Monograph 58: 1-102.

Richards, W.P. 1984. The ecology of tropical forest bryophytes. Pp. 12331270. In: Schuster, R.M. (Ed.). v.2. New Manual of Bryology. Japan, Hattori Botanical Laboratory.

Rocha, W.J.S.F.; Chaves, J.M.; Rocha, C.C.; Funch, L. \& Juncá, F.A. 2005. Avaliação Ecológica Rápida da Chapada Diamantina. Pp. 29-45. In: Juncá, F.A., Funch, L. \& Rocha, W. (Orgs.). Biodiversidade e Conservação da Chapada Diamantina. Ministério do Meio Ambiente.

Santos, N.D. \& Costa, D.P. 2010a. Altitudinal zonation of liverworts in the Atlantic Forest, Southeastern Brazil. The Bryologist 113(3): 631-645.

Santos, N. D. \& Costa, D.P. 2010b. Phytogeography of the liverwort flora of the Atlantic Forest of south-eastern Brazil. Journal of Bryology 32: 9-22.

Sharp, A.J.; Crum, H. \& Eckel, P. 1994. The moss flora of Mexico. Memoirs of The New York Botanical Garden 69: 1-1113.

Silva, M.P.P. \& Pôrto, K.C. 2009. Effect of fragmentation on the community structure of epixylic bryophytes in Atlantic Forest remnants in the Northeast of Brazil. Biodiversity and Conservation 18: 317-337.

Valente, E.B. \& Pôrto, K.C. 2006. Hepáticas (Marchantiophyta) de um fragmento de Mata Atlântica na Serra da Jibóia, município de Santa Terezinha, BA, Brasil. Acta Botanica Brasilica 20: 433-441.

Valente, E. B.; Pôrto, K. C. \& Bastos, C. J. P. 2011. Checklist of Bryophytes of Chapada Diamantina, Bahia, Brazil. Boletim do Instituto de Botânica 21: 111-124.

Valente, E.B.; Pôrto, K.C.; Bôas-Bastos, S.B.V. \& Bastos, C.J.P. 2009. Musgos (Bryophyta) de um fragmento de Mata Atlântica na Serra da Jibóia, município de Santa Terezinha, BA, Brasil. Acta Botanica Brasilica 23: 369-375.

Van Reenen, G.B.A. \& Gradstein, S.R. 1983. A transect analysis of the bryophyte vegetation along an altitudinal gradient on he Sierra Nevada de Santa Marta, Colombia. Acta Botania Neerlandica 32: 163-175.

Van Reenen, G.B.A. \& Gradstein, S.R. 1984. An investigation of bryophyte distribution and ecology along an altitudinal gradient in the Andes of Colombia. Journal of the Hattori Botanical Laboratory 56: 79-84.

Vanderpoorten, A. \& Goffinet, B. 2009. Introduction to Bryophytes. Cambridge University Press.

Veloso, H.P.; Rangel Filho, A.L.R. \& Lima, J.C. 1991. Classificação da vegetação brasileira, adaptada a um sistema universal. Rio de Janeiro: IBGE.

Velloso, A.L.; Sampaio, E.V.S.B. \& Pareyn, F.G.C. 2002. Ecorregiões propostas para o Bioma Caatinga. Recife, Associação Plantas do Nordeste, Instituto de Conservação Ambiental, The Nature Conservancy do Brasil.

Vilas Bôas-Bastos. S.B. \& Bastos, C.J.P. 2008. Neckeraceae (Bryophyta, Bryopsida) da Reserva Ecológica da Michelin, Igrapiúna, Bahia, Brasil. Sitientibus, Série Ciências Biológicas 8: 263-274.

Vilas Bôas-Bastos, S.B. \& Bastos, C.J.P. 1998. Briófitas de uma área de cerrado no município de Alagoinhas, Bahia, Brasil. Tropical Bryology 15: 101-110.

Visnadi, S.R. 2004. Distribuição da brioflora em diferentes fisionomias de cerrado da Reserva Biológica e Estação Experimental de Mogi-Guaçu, SP, Brasil. Acta Botanica Brasilica 18(4): 965-973.

Visnadi, S.R. 2005. Brioflora da Mata Atlântica do estado de São Paulo: região norte. Hoehnea 32(2): 215-231.

Visnadi, S. R. \& Vital, D. M. 2000. Lista das briófitas ocorrentes no Parque Estadual das Fontes do Ipiranga - PEFI. Hoehnea 27(3): 279-294.

Yano, O. 1981. A Checklist of Brazilian Mosses. Journal of the Hattori Botanical Laboratory 50: 279-456.

Yano, O. 1984. A Checklist of Brazilian Liverworts and Hornworts. Journal of the Hattori Botanical Laboratory 56: 481-548.

Yano, O. 1987. Briófitas. Pp: 11-12. In: Giulietti, A.M.; Menezes, N.L.; Pirani, J.R.; Meguro, M. \& Wanderley, M.G.L. (Org.). Flora da Serra do Cipó, Minas Gerais: caracterização e lista de espécies. São Paulo: Boletim de Botânica da Universidade de São Paulo.

Yano, O. 1989a. An Additional Checklist of Brazilian the Bryophytes. Journal of the Hattori Botanical Laboratory 66: 371-434.

Yano, O. 1989b. Briófitas. Pp.27-30 In: Fidalgo, O. \& Bononi, V.L.R. (Ed.) Técnicas de coleta, preservação e herborização de material botânico. Série Documentos/Instituto de Botânica, São Paulo. 
Yano, O. 1995. A New Additional Annotated Checklist of Brazilian Bryophytes. Journal of the Hattori Botanical Laboratory 78:137-182.

Yano, O. 1996a. A checklist of the Brazilian Bryophytes. Boletim do Instituto de Botânica 10: 47-232.

Yano, O. 2005. Adição às brióftas da Reserva Natural da Vale do Rio Doce, Linhares Espírito Santo, Brasil. Boletim do Museu de Biologia Mello Leitão 18: 15-58.

Yano, O. 2006. Novas adições ao catálogo de Briófitas Brasileiras. Boletim do Instituto de Botânica 17: 1-142.

Yano, O. 2008. Catálogo de Antóceros e Hepáticas Brasileiros: literatura original, basiônimo, localidade-tipo e distribuição geográfica. Boletim do Instituto de Botânica 19: 1-110.

Yano, O. \& Bastos, C.J.P. 1994. Musgos do estado da Bahia, Brasil. Biologica Brasilica 6: 9-26.
Yano, O. \& Carvalho, A. B. 1995. Briófitas da Serra da Piedade, Minas Gerais, Brasil. Pp. 15-25. In: Anais do 9oㅡㄹ Congresso da Sociedade Botânica de São Paulo.

Yano, O. \& Peralta, D.F. 2006. Briófitas coletadas por Daniel Moreira Vital no Estado da Bahia, Brasil. Boletim do Instituto de Botânica 18: 33-73.

Yano, O.; Pirani, J.R. \& Santos, D.P. 1985. O gênero Sphagnum (Bryopsida) nas regiões sul e sudeste do Brasil. Revista Brasileira de Botânica 8: $55-80$.

Yano, O. \& Peralta, D.F. 2009. Flora de Grão-Mogol, Minas Gerais: Briófitas (Bryophyta e Marchantiophyta). Boletim de Botânica da Universidade de São Paulo 27(1): 1-26.

Zander, R.H. 1993. Genera of the Pottiaceae: Mosses of Harsh environments. Bulletin of the Buffalo Society of Natural Sciences 32: 1-378. 